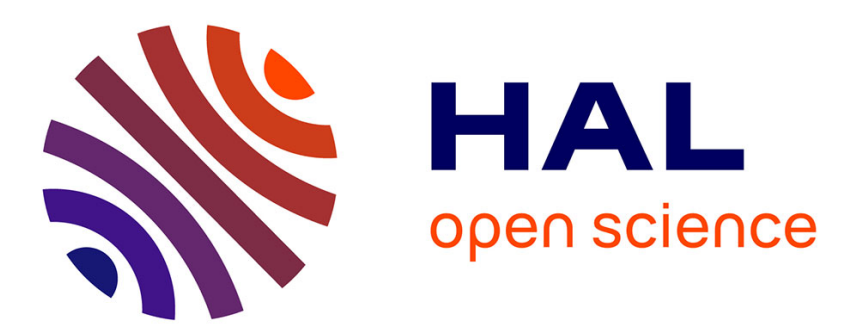

\title{
An entropy preserving relaxation scheme for ten-moments equations with source terms
}

\author{
Christophe Berthon, Bruno Dubroca, Afeintou Sangam
}

\section{To cite this version:}

Christophe Berthon, Bruno Dubroca, Afeintou Sangam. An entropy preserving relaxation scheme for ten-moments equations with source terms. Communications in Mathematical Sciences, 2015, Communications in Mathematical Sciences, 13 (8), pp. 2119-2154. 10.4310/CMS.2015.v13.n8.a7 . hal-01255069

\section{HAL Id: hal-01255069 \\ https://hal.inria.fr/hal-01255069}

Submitted on 15 Jan 2016

HAL is a multi-disciplinary open access archive for the deposit and dissemination of scientific research documents, whether they are published or not. The documents may come from teaching and research institutions in France or abroad, or from public or private research centers.
L'archive ouverte pluridisciplinaire HAL, est destinée au dépôt et à la diffusion de documents scientifiques de niveau recherche, publiés ou non, émanant des établissements d'enseignement et de recherche français ou étrangers, des laboratoires publics ou privés. 


\title{
AN ENTROPY PRESERVING RELAXATION SCHEME FOR TEN-MOMENTS EQUATIONS WITH SOURCE TERMS *
}

\author{
CHRISTOPHE BERTHON ${ }^{\dagger}$, BRUNO DUBROCA ${ }^{\ddagger}$, AND AFEINTOU SANGAM,
}

\begin{abstract}
The present paper concerns the derivation of finite volume methods to approximate weak solutions of Ten-Moments equations with source terms. These equations model compressible anisotropic flows. A relaxation-type scheme is proposed to approximate such flows. Both robustness and stability conditions of the suggested finite volume methods are established. To prove discrete entropy inequalities, we derive a new strategy based on local minimum entropy principle and never use some approximate PDE's auxiliary model as usually recommended. Moreover, numerical simulations in $1 \mathrm{D}$ and in $2 \mathrm{D}$ illustrate our approach.
\end{abstract}

Key words. Hyperbolic system, Ten-Moments equations, source terms, Godunov type schemes, discrete entropy inequalities, discrete entropy minimum principle.

AMS subject classifications. 65M60, 65M12.

\section{Introduction}

The main applications to be reached in this paper concern plasma physics. The physics under consideration belongs to Inertial Confinement Fusion (ICF). In this context a target capsule containing a fusible mixture is compressed and heated by laser beams. A dense plasma is then created by ionization of the fusible mixture. The interaction of the laser hot spots, the so-called speckles, with a coronal plasma created from the outer parts of the target, leads to an intense and inhomogeneous heating of electrons. Moreover, the electron distribution function acquires an anisotropy in the direction of the laser polarization. The resulting plasma model can be characterized by a non-isotropic pressure tensor.

Kinetic theories can accurately model a large number of particles. However, numerical computations of kinetic theories are, in general, resource consuming both in time and storage space, and are limited in a small computational domain of physical/velocities space. Kinetic descriptions provide a lot of information which are not accessible by experiment. Reversely, fluid models based on velocity moments can be simulated for a large time and a large domain. These fluid models, involving adequate closure procedures [26, 27], lead to important information that fit with experimental results $[5,18,29]$. The Ten-Moments system is a such significant fluid model that possesses a kinetic theory. It is the second hierarchy of symmetric hyperbolic systems of partial differential equations according to the moment closures procedure of Levermore $[26,27]$. The Ten-Moments equations are obtained as the Gaussian closure of Levermore

${ }^{*}$ The first author thank project ANR-12-IS01-0004-01 GEONUM to partially support this work. The third author thanks to CNRS and INRIA for 'délégations' grants during years 2011, 2012, 2013. The authors thank Lise-Marie IMBERT-GÉRARD at Courant Institute of Mathematical Sciences at New York for carefully improving the english of the paper.

${ }^{\dagger}$ Laboratoire de Mathématiques Jean Leray, CNRS UMR 6629, Université de Nantes, 2 rue de la Houssinière, BP 92208, 44322 Nantes, France, Christophe.Berthon@math.univ-nantes.fr

‡INSTITUT DE MATHÉMATIQUES DE BORDEAUX, CNRS UMR 5251, CENTRE LASERS INTENSES ET APPLICATIONS, CNRS UMR 5107, UNIVERSITÉ BORDEAUX 1, 351, COURS DE LA LIBÉRATION, 33405 TALENCE CEDEX, FRANCE, BRUNO.DUBROCA@MATH.UBORDEAUX1.FR

§Laboratoire J.A. Dieudonné, CNRS UMR 7351, Université Nice Sophia Antipolis, Parc Valrose 06108 Nice France, and INRIA Sophia Antipolis 2004, Route des Lucioles, BP 93, 06902 Sophia Antipolis Cedex, France, Afeintou.Sangam@unice.fr 
method. It takes into account the anisotropy of the particles distribution function and is well-suited for numerical computations of transition regimes.

Following the work of Levermore [26, 27] (see also [10, 19, 18, 29]), the adequate model to describe such anisotropic flows is governed by the Ten-Moments system with appropriate source terms. The present study is devoted to the numerical approximation of the weak solutions of the hyperbolic system extracted from Ten-Moments model combined with relevant source terms describing the interaction of matter with laser light. During the last decade several numerical strategies have been proposed to capture the main properties of hyperbolic part of the underlined Ten-Moments model. For instance either Roe scheme in [10, 19] or HLLE scheme in [19] have been designed for this purpose. Recently in the plasma physics framework, robust numerical schemes have been developed to approximate the Ten-Moments equations [18, 5, 31]. Numerical approximations in this context are based on splitting strategy: the hyperbolic part of the Ten-Moments model is first solved by a convenient scheme, the other terms of the model are then treated as source terms. Great efforts have been hence put on the derivation of approximations of the hyperbolic part of the model. Regarding the numerical stability of schemes devoted to this hyperbolic part, discrete entropy inequalities have been established in [5]. The derivation of discrete entropy inequalities is based on relaxation techniques, previously introduced in the context of Euler equations and/or Saint-Venant system $[3,8, ?, 12]$. More precisely, the entropy preserving property comes from a suitable use of relaxation models where stiff source terms play a crucial role.

Here, we first construct a scheme dedicated to approximate the hyperbolic part of the Ten-Moments model. The main novelty stays in the considered source term which involves a gradient potential $[11,14,36]$. This scheme is a relaxation type numerical method $[3,8,9,12]$. Next, we prove that the proposed scheme is entropy preserving. Let us emphasize that the discrete inequalities are established by arguing a suitable extension of the technique introduced in [7]. Put in other words, at the discrepancy with the previous works $[3,8,9,12]$, the stiff relaxation source term is not evaluated in the proposed discrete entropy inequalities.

This work is organized as follows. In the next section, we present the Ten-Moments model with adequate source terms and we exhibit the entropy inequalities. For the sake of completeness, in Section 3 we introduce the main notations to derive a Godunov-type scheme [21]. A particular attention is paid to the rotational invariance, and we prove that a suitable 1D scheme will give a robust and stable 2D numerical approximation. A relaxation model is proposed in Section 4 while a relaxation scheme is derived in Section 5. Section 6 establishes the robustness and stability statement. Numerical experiments are performed in Section 7 to illustrate both accuracy and robustness of the proposed numerical scheme in 1D. Two dimensional realistic simulations are also displayed. A conclusion is given in the last section.

\section{Main Properties of the Mathematical Model}

The Ten-Moments equations describe anisotropic flows arising from either gas dynamics $[10,19,26,27]$ or plasma physics $[5,18,29,31]$. These flows depend on a preferential direction of the fluid motion. The $2 \mathrm{D}$ model of such flows in the context of plasma physics, taking into account the laser-matter interaction, is given as follows

$$
\left\{\begin{array}{l}
\partial_{t} \rho+\nabla \cdot(\rho \boldsymbol{u})=0, \\
\partial_{t}(\rho \boldsymbol{u})+\boldsymbol{\nabla} \cdot(\rho \boldsymbol{u} \otimes \boldsymbol{u}+\boldsymbol{P})=-\frac{1}{2} \rho \boldsymbol{\nabla} W, \\
\partial_{t}(\rho \boldsymbol{u} \otimes \boldsymbol{u}+\boldsymbol{P})+\boldsymbol{\nabla} \cdot(\rho \boldsymbol{H} \otimes \boldsymbol{u})^{S}=-(\rho \boldsymbol{\nabla} W \otimes \boldsymbol{u})^{S},
\end{array}\right.
$$


with $\rho>0$ the density of matter, $\boldsymbol{u}=\left(u_{1} u_{2} 0\right)^{T} \in \mathbb{R}^{3}$ the flow velocity and $\boldsymbol{P}$ the matrix of pressure. Let us introduce the matrix $\boldsymbol{E}=\rho \boldsymbol{u} \otimes \boldsymbol{u}+\boldsymbol{P}$ to define the matrix of total energy per unit volume. For the sake of simplicity in the notations, let us set $\varepsilon=\frac{1}{\rho} \boldsymbol{P}$ the internal energy tensor. The matrix $\boldsymbol{H}=\boldsymbol{u} \otimes \boldsymbol{u}+3 \boldsymbol{P} / \rho$ is the generalized enthalpy per unit mass. The components of the second rank tensor, $(\rho \boldsymbol{\nabla} W \otimes \boldsymbol{u})^{S}$ are given by:

$$
(\rho \nabla W \otimes \boldsymbol{u})^{S}=\frac{1}{2}(\rho \nabla W \otimes \boldsymbol{u}+\rho \boldsymbol{u} \otimes \nabla W) .
$$

The components of the third rank tensor, $(\rho \boldsymbol{H} \otimes \boldsymbol{u})^{S}$ are given by:

$$
(\rho \boldsymbol{H} \otimes \boldsymbol{u})_{i j k}^{S}=\frac{1}{3}\left(\rho \boldsymbol{H}_{i j} \boldsymbol{u}_{k}+\rho \boldsymbol{H}_{j k} \boldsymbol{u}_{i}+\rho \boldsymbol{H}_{k i} \boldsymbol{u}_{j}\right) .
$$

The given function $W:=W(x, y, t)$ is the electron quiver energy in the laser light $[18,23$, $29,30,31]$. The reader is referred to [31] for details on the full system. The model (2.1) can be rewritten as

$$
\partial_{t} \mathcal{U}+\partial_{x} \mathcal{F}(\mathcal{U})+\partial_{y} \mathcal{G}(\mathcal{U})=\mathcal{S}\left(\mathcal{U},\left(\boldsymbol{e}_{1} \otimes \boldsymbol{e}_{1}\right) \nabla W\right)+\mathcal{T}\left(\mathcal{U},\left(\boldsymbol{e}_{2} \otimes \boldsymbol{e}_{2}\right) \nabla W\right)
$$

where

$$
\begin{aligned}
& \mathcal{U}=\left(\begin{array}{c}
\rho \\
\rho u_{1} \\
\rho u_{2} \\
\rho u_{1}^{2}+\rho \varepsilon_{11} \\
\rho u_{1} u_{2}+\rho \varepsilon_{12} \\
\rho u_{2}^{2}+\rho \varepsilon_{22} \\
\rho \varepsilon_{33}
\end{array}\right), \mathcal{F}(\mathcal{U})=\left(\begin{array}{c}
\rho u_{1} \\
\rho u_{1}^{2}+\rho \varepsilon_{11} \\
\rho u_{1} u_{2}+\rho \varepsilon_{12} \\
\rho u_{1}^{3}+3 \rho \varepsilon_{11} u_{1} \\
\rho u_{1}^{2} u_{2}+\rho \varepsilon_{11} u_{2}+2 \rho \varepsilon_{12} u_{1} \\
\rho u_{1} u_{2}^{2}+2 \rho \varepsilon_{12} u_{2}+\rho \varepsilon_{22} u_{1} \\
\rho \varepsilon_{33} u_{1}
\end{array}\right) \\
& \mathcal{G}(\mathcal{U})=\left(\begin{array}{c}
\rho u_{2} \\
\rho u_{1} u_{2}+\rho \varepsilon_{12} \\
\rho u_{2}^{2}+\rho \varepsilon_{22} \\
\rho u_{1}^{2} u_{2}+\rho \varepsilon_{11} u_{2}+2 \rho \varepsilon_{12} u_{1} \\
\rho u_{1} u_{2}^{2}+2 \rho \varepsilon_{12} u_{2}+\rho \varepsilon_{22} u_{1} \\
\rho u_{2}^{3}+3 \rho \varepsilon_{22} u_{2} \\
\rho \varepsilon_{33} u_{2}
\end{array}\right), \mathcal{S}\left(\mathcal{U},\left(\boldsymbol{e}_{1} \otimes \boldsymbol{e}_{1}\right) \nabla W\right)=\left(\begin{array}{c}
0 \\
-\frac{1}{2} \rho \partial_{x} W \\
0 \\
-\rho u_{1} \partial_{x} W \\
-\frac{1}{2} \rho u_{2} \partial_{x} W \\
0 \\
0
\end{array}\right) \\
& \mathcal{T}\left(\mathcal{U},\left(\boldsymbol{e}_{2} \otimes \boldsymbol{e}_{2}\right) \nabla W\right)=\left(\begin{array}{c}
0 \\
0 \\
-\frac{1}{2} \rho \partial_{y} W \\
0 \\
-\frac{1}{2} \rho u_{1} \partial_{y} W \\
-\rho u_{2} \partial_{y} W \\
0
\end{array}\right)
\end{aligned}
$$

with $\boldsymbol{e}_{1}=\left(\begin{array}{lll}1 & 0 & 0\end{array}\right)^{T}$ and $\boldsymbol{e}_{2}=\left(\begin{array}{lll}0 & 1 & 0\end{array}\right)^{T}$.

The state vector $\mathcal{U}$ belongs to the so-called set of the physically admissible states defined as follows:

$$
\Omega=\left\{\mathcal{U}=(\rho \rho \boldsymbol{u} \boldsymbol{E})^{T} / \rho>0, \rho \boldsymbol{\varepsilon}=\boldsymbol{E}-\rho \boldsymbol{u} \otimes \boldsymbol{u} \text { verifying } \boldsymbol{\xi}^{T} \cdot \rho \boldsymbol{\varepsilon} \cdot \boldsymbol{\xi}>0 \forall \boldsymbol{\xi} \in \mathbb{R}^{3}\right\} .
$$


Let us notice that the space $\Omega$ is a closed convex cone.

For simplicity in notations, we propose to introduce a directional flux function defined as follows:

$$
\mathbb{F}(\mathcal{U}, \boldsymbol{n})=(\boldsymbol{u} \cdot \boldsymbol{n}) \mathcal{U}+\left(\begin{array}{c}
0 \\
\rho \varepsilon \boldsymbol{n} \\
2((\rho \varepsilon \boldsymbol{n}) \otimes \boldsymbol{u})^{S}
\end{array}\right)
$$

where $\boldsymbol{n}$ denotes an arbitrary unit vector and the symmetric tensor $2((\rho \boldsymbol{\varepsilon} \boldsymbol{n}) \otimes \boldsymbol{u})^{S}$ is defined by

$$
2((\rho \boldsymbol{\varepsilon} \boldsymbol{n}) \otimes \boldsymbol{u})^{S}=(\rho \boldsymbol{\varepsilon} \boldsymbol{n}) \otimes \boldsymbol{u}+\boldsymbol{u} \otimes(\rho \varepsilon \boldsymbol{n}) .
$$

A directional source term is also introduced through

$$
\mathbb{S}(\mathcal{U}, \boldsymbol{\nabla} W, \boldsymbol{n})=\left(\begin{array}{c}
0 \\
-\frac{1}{2} \rho(\boldsymbol{n} \otimes \nabla W) \boldsymbol{n} \\
-\rho(((\boldsymbol{n} \otimes \nabla W) \boldsymbol{n}) \otimes \boldsymbol{u}
\end{array}\right) .
$$

Involving such notations, we remark that

$$
\left\{\begin{array}{l}
\mathcal{F}(\mathcal{U})=\mathbb{F}\left(\mathcal{U}, \boldsymbol{e}_{1}\right), \text { and } \mathcal{G}(\mathcal{U})=\mathbb{F}\left(\mathcal{U}, \boldsymbol{e}_{2}\right), \\
\mathcal{S}\left(\mathcal{U},\left(\boldsymbol{e}_{1} \otimes \boldsymbol{e}_{1}\right) \nabla W\right)=\mathbb{S}\left(\mathcal{U}, \nabla W, \boldsymbol{e}_{1}\right), \text { and } \mathcal{T}\left(\mathcal{U},\left(\boldsymbol{e}_{2} \otimes \boldsymbol{e}_{2}\right) \nabla W\right)=\mathbb{S}\left(\mathcal{U}, \nabla W, \boldsymbol{e}_{2}\right)
\end{array}\right.
$$

Therefore the model (2.1) reads

$$
\partial_{t} \mathcal{U}+\partial_{x} \mathbb{F}\left(\mathcal{U}, \boldsymbol{e}_{1}\right)+\partial_{y} \mathbb{F}\left(\mathcal{U}, \boldsymbol{e}_{2}\right)=\mathbb{S}\left(\mathcal{U}, \nabla W, \boldsymbol{e}_{1}\right)+\mathbb{S}\left(\mathcal{U}, \nabla W, \boldsymbol{e}_{2}\right)
$$

One of main advantages of considering a homogeneous directional flux and a directional source term functions $\mathbb{F}$ and $\mathbb{S}$ respectively, is an easy establishment of the rotational invariance satisfied by system (2.2)-(2.5) (see Appendix B). Indeed, let us consider the following rotational operator defined by

$$
\bar{R}(\mathcal{U})=\left(\begin{array}{c}
\rho \\
\rho R \boldsymbol{u} \\
\rho R(\boldsymbol{u} \otimes \boldsymbol{u}+\varepsilon) R^{T}
\end{array}\right)
$$

where $R$ stands for a rotational matrix given, for all $\theta \in(0,2 \pi)$ by,

$$
R=\left(\begin{array}{ccc}
\cos \theta & -\sin \theta & 0 \\
\sin \theta & \cos \theta & 0 \\
0 & 0 & 1
\end{array}\right)
$$

Then we have the following rotational invariance property satisfied by the flux and the source term functions (see Appendix A):

$$
\begin{aligned}
& \mathbb{F}(\bar{R}(\mathcal{U}), R \boldsymbol{n})=\bar{R}(\mathbb{F}(\mathcal{U}, \boldsymbol{n})), \\
& \mathbb{S}(\bar{R}(\mathcal{U}), R \boldsymbol{\nabla} W, R \boldsymbol{n})=\bar{R}(\mathbb{S}(\mathcal{U}, \boldsymbol{\nabla} W, \boldsymbol{n})),
\end{aligned}
$$


where the quantities $\mathbb{S}(\bar{R}(\mathcal{U}), \nabla W, R \boldsymbol{n})$ and $\bar{R}(\mathbb{S}(\mathcal{U}, \boldsymbol{\nabla} W, \boldsymbol{n}))$ read

$$
\begin{aligned}
& \mathbb{S}(\bar{R}(\mathcal{U}), R \boldsymbol{\nabla} W, R \boldsymbol{n})=\left(\begin{array}{c}
0 \\
-\frac{1}{2} \rho(R \boldsymbol{n} \otimes R \boldsymbol{\nabla} W) R \boldsymbol{n} \\
-\rho(((R \boldsymbol{n} \otimes R \boldsymbol{\nabla} W) R \boldsymbol{n}) \otimes R \boldsymbol{u})^{S}
\end{array}\right), \\
& \bar{R}(\mathbb{S}(\mathcal{U}, \boldsymbol{\nabla} W, \boldsymbol{n}))=\left(\begin{array}{c}
0 \\
-\frac{1}{2} \rho R((\boldsymbol{n} \otimes \boldsymbol{\nabla} W) \boldsymbol{n}) \\
-\rho R(((\boldsymbol{n} \otimes \boldsymbol{\nabla} W) \boldsymbol{n}) \otimes \boldsymbol{u})^{S} R^{T}
\end{array}\right) .
\end{aligned}
$$

This rotational invariance property of the flux and the source term functions (2.10) can be supplemented by the following additional ones.

LEMMA 2.1. The set of admissible states $\Omega$ is invariant by the rotational operator $\bar{R}$. Moreover, we have, for all $\mathcal{U} \in \Omega$,

$$
\operatorname{det}(\varepsilon(\bar{R}(\mathcal{U})))=\operatorname{det}(\varepsilon(\mathcal{U})) .
$$

Proof. By definition of $\bar{R}$, we have $\bar{R}(\Omega)=\Omega$. Indeed, the matrix $R \varepsilon R^{T}$ is positive definite. Now, since $\varepsilon(\bar{R}(\mathcal{U}))=R \varepsilon(\mathcal{U}) R^{T}$, the determinant is preserved.

We next focus on the algebraic properties of (2.2)-(2.5), omitting the source terms $\mathcal{S}\left(\mathcal{U},\left(\boldsymbol{e}_{1} \otimes \boldsymbol{e}_{1}\right) \boldsymbol{\nabla} W\right)$ and $\mathcal{T}\left(\mathcal{U},\left(\boldsymbol{e}_{2} \otimes \boldsymbol{e}_{2}\right) \boldsymbol{\nabla} W\right)$. The proof of the following result is detailed in $[10,19,26]$.

LEMma 2.2. The system (2.2)-(2.5) without the source terms $\mathcal{S}\left(\mathcal{U},\left(\boldsymbol{e}_{1} \otimes \boldsymbol{e}_{1}\right) \nabla W\right)$ and $\mathcal{T}\left(\mathcal{U},\left(\boldsymbol{e}_{2} \otimes \boldsymbol{e}_{2}\right) \nabla W\right)$

$$
\partial_{t} \mathcal{U}+\partial_{x} \mathcal{F}(\mathcal{U})+\partial_{y} \mathcal{G}(\mathcal{U})=0
$$

is hyperbolic and its eigenvalues along the unit vector $\boldsymbol{n}=\left(n_{1} n_{2} 0\right)^{T}$ are given by

$$
\Lambda(\mathcal{U}, \boldsymbol{n})=\left\{\boldsymbol{u} \cdot \boldsymbol{n}-c, \boldsymbol{u} \cdot \boldsymbol{n}-\frac{c}{\sqrt{3}}, \boldsymbol{u} \cdot \boldsymbol{n}, \boldsymbol{u} \cdot \boldsymbol{n}, \boldsymbol{u} \cdot \boldsymbol{n}, \boldsymbol{u} \cdot \boldsymbol{n}+\frac{c}{\sqrt{3}}, \boldsymbol{u} \cdot \boldsymbol{n}+c\right\},
$$

where $c=\sqrt{3 \boldsymbol{n}^{T} \cdot \boldsymbol{P} \cdot \boldsymbol{n} / \rho}$ is the sound speed.

Moreover, the characteristic fields associated to the eigenvalues $\boldsymbol{u} \cdot \boldsymbol{n} \pm c$ are genuinely nonlinear whereas the characteristic fields associated to the eigenvalues $\boldsymbol{u} \cdot \boldsymbol{n}, \boldsymbol{u} \cdot \boldsymbol{n} \pm$ $c / \sqrt{3}$ are linearly degenerated.

Furthermore, in order to rule out unphysical discontinuous solutions [24] of this hyperbolic part of $(2.2)-(2.5)$, the system is endowed with entropy inequalities. In fact the entropies for the hyperbolic part of (2.2)-(2.5) are compatible with classical solutions of the full system. Indeed the source terms neither produce nor dissipate entropies. More precisely, according to [26], the following statement can be established: LEMMA 2.3. The smooth solutions of system (2.2)-(2.5) satisfy the following non-trivial transport equations,

$$
\partial_{t} s(\varepsilon, v)+u_{1} \partial_{x} s(\varepsilon, v)+u_{2} \partial_{y} s(\varepsilon, v)=0
$$


where $s(\varepsilon, v)$ stands for the specific entropy given by

$$
s(\varepsilon, v)=\ln \left(\frac{\operatorname{det} \varepsilon}{\rho^{2}}\right)=\ln \left(v^{2} \operatorname{det} \varepsilon\right),
$$

with $v=1 / \rho$ the specific volume.

As a consequence, for all smooth functions $F: \mathbb{R} \rightarrow \mathbb{R}$, the smooth solutions of system (2.2)-(2.5) verify the following conservation law:

$$
\partial_{t} \rho F(s(\varepsilon, v))+\partial_{x} \rho F(s(\varepsilon, v)) u_{1}+\partial_{y} \rho F(s(\varepsilon, v)) u_{2}=0 .
$$

The function $\mathcal{U} \mapsto \rho F(s(\varepsilon, v))$ is convex if

$$
F^{\prime}(y)<0, \quad-\frac{1}{5} F^{\prime}(y)+F^{\prime \prime}(y)>0 .
$$

Then, the weak solutions of (2.2)-(2.5) satisfy the following entropy inequality:

$$
\partial_{t} \rho F(s(\varepsilon, v))+\partial_{x} \rho F(s(\varepsilon, v)) u_{1}+\partial_{y} \rho F(s(\varepsilon, v)) u_{2} \leq 0 .
$$

From now on, let us note that the specific entropy remains invariant by the rotational operator $(2.9), \bar{R}$. Indeed, by applying Lemma 2.1 , we immediately have

$$
s(\bar{R}(\mathcal{U}))=s(\mathcal{U}) .
$$

According to the Tadmor minimum principle [33], let us set up a minimum principle satisfied by the specific entropy.

LEMma 2.4. Let $\mathcal{U}$ be an entropy solution of (2.2)-(2.5), the following estimate holds

$$
\underset{\sqrt{x^{2}+y^{2}} \leq R}{\operatorname{Ess} \operatorname{Inf}} s(\varepsilon, v)(x, y, t) \leq \underset{\sqrt{x^{2}+y^{2}} \leq R+t u_{\max }}{\operatorname{Ess} \operatorname{Inf}} s(\varepsilon, v)(x, y, t=0),
$$

where $u_{\max }$ denotes the maximal speed of the fluid in the domain.

Proof. The proof follows the ideas introduced in [33]. First, assume the function $F$ defined in Lemma 2.3 satisfies (2.16) and is nonnegative, then the following relation can be established:

$$
\begin{aligned}
\int_{\sqrt{x^{2}+y^{2}} \leq R} \rho(x, y, t) & F(s(\varepsilon, v)(x, y, t)) d x d y \\
\leq & \int_{\sqrt{x^{2}+y^{2}} \leq R+t u_{\max }} \rho(x, y, t) F(s(\varepsilon, v)(x, y, t=0)) d x d y .
\end{aligned}
$$

To address such an issue, we introduce the truncated cone $C=\left\{\sqrt{x^{2}+y^{2}} \leq R+(t-\right.$ $\left.\tau) u_{\max }, 0 \leq \tau \leq t\right\}$. We denote $\left(n_{1} n_{2} n_{t}\right)^{T}$ the unit outward normal. Integrating the entropy inequality (2.17), the Green's Theorem gives

$$
\int_{\partial C} \rho(x, y, t) F(s(\varepsilon, v)(x, y, t))\left(n_{t}+n_{1} u_{1}+n_{2} u_{2}\right) d x d y d t \leq 0,
$$

which yields

$$
\begin{aligned}
& \iint_{\sqrt{x^{2}+y^{2}} \leq R} \rho(x, y, t) F(s(\varepsilon, v)(x, y, t)) d x d y \\
& -\int_{\sqrt{x^{2}+y^{2}} \leq R+t u_{\max }} \rho(x, y, t) F(s(\varepsilon, v)(x, y, t=0)) d x d y \\
& \leq-\int_{\text {oblique sides }} \rho(x, y, t) F(s(\varepsilon, v)(x, y, t))\left(n_{t}+n_{1} u_{1}+n_{2} u_{2}\right) d x d y d t .
\end{aligned}
$$


Now let us note that, on both oblique sides, we have

$$
n_{t}+n_{1} u_{1}+n_{2} u_{2} \geq 0
$$

so that the required inequality $(2.20)$ holds as soon as the function $F$ is nonnegative.

Next, let us choose a relevant definition for the function $F$ as follows,

$$
\begin{aligned}
& F(s(\varepsilon, v)(x, y, t))=\max \left(s_{0}-s(\varepsilon, v)(x, y, t), 0\right), \\
& s_{0}=\underset{\sqrt{x^{2}+y^{2}} \leq R+t u_{\max }}{\operatorname{Ess} \operatorname{Inf}} s(\varepsilon, v)(x, y, t=0),
\end{aligned}
$$

to obtain

$$
\max \left(s_{0}-s(\varepsilon, v)(x, y, t=0), 0\right)=0 \quad \text { a.e. }
$$

As a consequence, we get

$$
\int_{\sqrt{x^{2}+y^{2}} \leq R} \rho(x, y, t) \max \left(s_{0}-s(\varepsilon, v)(x, y, t), 0\right) d x d y \leq 0,
$$

and the proof is ended.

\section{Numerical Approximation}

Our purpose now is to derive a numerical scheme able to preserve robustness, discrete entropy inequalities and a minimum entropy principle. We consider a structured mesh in space and time made of space cells $I_{i, j}=\left(x_{i-1 / 2}, x_{i+1 / 2}\right) \times\left(y_{j-1 / 2}, y_{j+1 / 2}\right)$, and time intervals $\left(t^{n}, t^{n+1}\right)$, where

$$
\left\{\begin{array}{l}
x_{i+1 / 2}=(i+1 / 2) \Delta x, y_{j+1 / 2}=(j+1 / 2) \Delta y, \forall(i, j) \in \mathbb{Z}^{2}, \\
t^{n+1}=t^{n}+\Delta t, \forall n \in \mathbb{N}
\end{array}\right.
$$

with $\Delta x, \Delta y$ and $\Delta t$ the constant space and time increments. The time step $\Delta t$ will be restricted according to a CFL-like condition which will be detailed later on.

As usual, at a time $t^{n}$, a piecewise constant approximation of the solution is given,

$$
\mathcal{U}^{\Delta}\left(x, y, t^{n}\right)=\mathcal{U}_{i, j}^{n},(x, y) \in I_{i, j}
$$

To evolve in time this approximation, we adopt a directional splitting strategy. From now on, arguing the rotational invariance of the system (2.2)-(2.5), let us underline that a 1D scheme can be considered. Indeed, let us consider the following 1D scheme

$$
\mathcal{U}_{i}^{n+1}=\mathcal{U}_{i}^{n}-\frac{\Delta t}{\Delta x}\left(\widehat{\mathbb{F}}\left(\mathcal{U}_{i}^{n}, \mathcal{U}_{i+1}^{n}, \boldsymbol{e}_{1}\right)-\widehat{\mathbb{F}}\left(\mathcal{U}_{i-1}^{n}, \mathcal{U}_{i}^{n}, \boldsymbol{e}_{1}\right)\right)+\Delta t \widehat{\mathbb{S}}\left(\mathcal{U}_{i}^{n}, \nabla W, \boldsymbol{e}_{1}\right),
$$

to approximate the weak solutions of the 1D Ten-Moments model with source term given by

$$
\partial_{t} \mathcal{U}+\partial_{x} \mathbb{F}\left(\mathcal{U}, e_{1}\right)=\mathbb{S}\left(\mathcal{U}, \nabla W, e_{1}\right)
$$

Let us introduce the following CFL condition:

$$
\frac{\Delta t}{\Delta x} \max _{\lambda \in \Lambda_{1}}|\lambda| \leq \frac{1}{2}
$$


where we have set $\Lambda_{1}=\Lambda\left(\mathcal{U}_{i-1}^{n}, \boldsymbol{e}_{1}\right) \cup \Lambda\left(\mathcal{U}_{i}^{n}, \boldsymbol{e}_{1}\right) \cup \Lambda\left(\mathcal{U}_{i+1}^{n}, \boldsymbol{e}_{1}\right)$ to denote the set of all the eigenvalues associated to $\mathcal{U}_{i-1}^{n}, \mathcal{U}_{i}^{n}$ and $\mathcal{U}_{i+1}^{n}$ in the $\boldsymbol{e}_{1}$-direction (see (2.12)). Now, we assume that the above $1 \mathrm{D}$ scheme is robust; namely $\mathcal{U}_{i}^{n} \in \Omega$ implies $\mathcal{U}_{i}^{n+1} \in \Omega$.

Moreover, we impose the scheme to be entropy preserving:

$$
\rho_{i}^{n+1} F\left(s_{i}^{n+1}\right) \leq \rho_{i}^{n} F\left(s_{i}^{n}\right)-\frac{\Delta t}{\Delta x}\left(\widehat{\mathbb{G}}\left(\mathcal{U}_{i}^{n}, \mathcal{U}_{i+1}^{n}, \boldsymbol{e}_{1}\right)-\widehat{\mathbb{G}}\left(\mathcal{U}_{i-1}^{n}, \mathcal{U}_{i}^{n}, \boldsymbol{e}_{1}\right)\right),
$$

where $F$ satisfies $(2.16)$ and $\widehat{\mathbb{G}}\left(\mathcal{U}_{l}, \mathcal{U}_{r}, \boldsymbol{n}\right)$ is consistent with the entropy directional flux function:

$$
\widehat{\mathbb{G}}(\mathcal{U}, \mathcal{U}, \boldsymbol{n})=\rho F(s) \boldsymbol{u} \cdot \boldsymbol{n},
$$

and $s_{i}^{n}=s\left(\varepsilon_{i}^{n}, v_{i}^{n}\right)$ with $s$ defined by $(2.14)$.

In addition, we assume that a minimum entropy principle is satisfied by the 1D scheme (3.1) as follows:

$$
s_{i}^{n+1} \geq \min \left(s_{i-1}^{n}, s_{i}^{n}, s_{i+1}^{n}\right) .
$$

Equipped with the above 1D scheme, we now adopt the following $2 \mathrm{D}$ extension:

$$
\begin{aligned}
\mathcal{U}_{i, j}^{n+1}=\mathcal{U}_{i, j}^{n} & -\frac{\Delta t}{\Delta x}\left(\widehat{\mathbb{F}}\left(\mathcal{U}_{i, j}^{n}, \mathcal{U}_{i+1, j}^{n}, \boldsymbol{e}_{1}\right)-\widehat{\mathbb{F}}\left(\mathcal{U}_{i-1, j}^{n}, \mathcal{U}_{i, j}^{n}, \boldsymbol{e}_{1}\right)\right) \\
& -\frac{\Delta t}{\Delta y}\left(\widehat{\mathbb{F}}\left(\mathcal{U}_{i, j}^{n}, \mathcal{U}_{i, j+1}^{n}, \boldsymbol{e}_{2}\right)-\widehat{\mathbb{F}}\left(\mathcal{U}_{i, j-1}^{n}, \mathcal{U}_{i, j}^{n}, \boldsymbol{e}_{2}\right)\right) \\
& +\Delta t \widehat{\mathbb{S}}\left(\mathcal{U}_{i, j}^{n}, \nabla W, \boldsymbol{e}_{1}\right)+\Delta t \widehat{\mathbb{S}}\left(\mathcal{U}_{i, j}^{n}, \nabla W, \boldsymbol{e}_{2}\right),
\end{aligned}
$$

The time step is here restricted according to a half-CFL condition given by

$$
\max _{\lambda \in \Lambda_{1} \cup \Lambda_{2}}\left(\frac{\Delta t}{\Delta x}|\lambda|, \frac{\Delta t}{\Delta y}|\lambda|\right) \leq \frac{1}{4},
$$

where $\Lambda_{1}=\Lambda\left(\mathcal{U}_{i-1, j}^{n}, \boldsymbol{e}_{1}\right) \cup \Lambda\left(\mathcal{U}_{i, j}^{n}, \boldsymbol{e}_{1}\right) \cup \Lambda\left(\mathcal{U}_{i+1, j}^{n}, \boldsymbol{e}_{1}\right)$ denotes the set of all the eigenvalues associated to $\mathcal{U}_{i-1, j}^{n}, \mathcal{U}_{i, j}^{n}$ and $\mathcal{U}_{i+1, j}^{n}$ in the $\boldsymbol{e}_{1}$-direction, and where $\Lambda_{2}=\Lambda\left(\mathcal{U}_{i, j-1}^{n}, \boldsymbol{e}_{2}\right) \cup$ $\Lambda\left(\mathcal{U}_{i, j}^{n}, \boldsymbol{e}_{2}\right) \cup \Lambda\left(\mathcal{U}_{i, j+1}^{n}, \boldsymbol{e}_{2}\right)$ denotes the set of all the eigenvalues associated to $\mathcal{U}_{i-1, j}^{n}, \mathcal{U}_{i, j}^{n}$ and $\mathcal{U}_{i+1, j}^{n}$ in the $\boldsymbol{e}_{2}$-direction.

Nowadays, we establish that the 2D scheme (3.5) preserves the required robustness and stability properties.

THEOREM 3.1. We suppose that, for all rotational operator $\bar{R}$ defined by (2.9), the numerical flux function and the discretization of the source term, introduced in (3.1), are rotational invariant:

$$
\left\{\begin{array}{l}
\widehat{\mathbb{F}}\left(\bar{R}\left(\mathcal{U}_{i}^{n}\right), \bar{R}\left(\mathcal{U}_{i+1}^{n}\right), R \boldsymbol{n}\right)=\bar{R}\left(\widehat{\mathbb{F}}\left(\mathcal{U}_{i}^{n}, \mathcal{U}_{i+1}^{n}, \boldsymbol{n}\right)\right) \\
\widehat{\mathbb{S}}\left(\bar{R}\left(\mathcal{U}_{i}^{n}\right), R \boldsymbol{\nabla} W, R \boldsymbol{n}\right)=\bar{R} \widehat{\mathbb{S}}\left(\mathcal{U}_{i}, \boldsymbol{\nabla} W, \boldsymbol{n}\right)
\end{array}\right.
$$

and the numerical entropy flux function satisfies

$$
\widehat{\mathbb{G}}\left(\bar{R}\left(\mathcal{U}_{i}^{n}\right), \bar{R}\left(\mathcal{U}_{i+1}^{n}\right), R \boldsymbol{n}\right)=\widehat{\mathbb{G}}\left(\mathcal{U}_{i}^{n}, \mathcal{U}_{i+1}^{n}, \boldsymbol{n}\right) .
$$

Then the 2D scheme (3.5), under the half-CFL condition (3.6), satisfies the following properties:

i) robustness: $\mathcal{U}_{i, j}^{n} \in \Omega$ implies $\mathcal{U}_{i, j}^{n+1} \in \Omega$, 
ii) entropy preservation:

$$
\begin{aligned}
\rho_{i, j}^{n+1} F\left(s_{i, j}^{n+1}\right) \leq \rho_{i, j}^{n} F\left(s_{i, j}^{n}\right) & -\frac{\Delta t}{\Delta x}\left(\widehat{\mathbb{G}}\left(\mathcal{U}_{i, j}^{n}, \mathcal{U}_{i+1, j}^{n}, \boldsymbol{e}_{1}\right)-\widehat{\mathbb{G}}\left(\mathcal{U}_{i-1, j}^{n}, \mathcal{U}_{i, j}^{n}, \boldsymbol{e}_{1}\right)\right) \\
& -\frac{\Delta t}{\Delta y}\left(\widehat{\mathbb{G}}\left(\mathcal{U}_{i, j}^{n}, \mathcal{U}_{i, j+1}^{n}, \boldsymbol{e}_{2}\right)-\widehat{\mathbb{G}}\left(\mathcal{U}_{i, j-1}^{n}, \mathcal{U}_{i, j}^{n}, \boldsymbol{e}_{2}\right)\right),
\end{aligned}
$$

where $s_{i, j}^{n}=s\left(\varepsilon_{i, j}^{n}, v_{i, j}^{n}\right)$ with $s$ defined by $(2.14)$,

iii) minimum entropy principle preservation:

$$
s_{i, j}^{n+1} \geq \min \left(s_{i-1, j}^{n}, s_{i, j-1}^{n}, s_{i, j}^{n}, s_{i+1, j}^{n}, s_{i, j+1}^{n}\right) .
$$

Proof. The establishment of the three statements comes from a reformulation of the 2D schemes (3.5) as a convex combination of the associated 1D scheme.

First, we exhibit the required convex combination satisfied by (3.5). To address such an issue, let us introduce the following updated state given by,

$$
\begin{aligned}
\mathcal{U}_{i, j}^{x}=\mathcal{U}_{i, j}^{n} & -\frac{\Delta t}{\Delta x / 2}\left(\widehat{\mathbb{F}}\left(\mathcal{U}_{i, j}^{n}, \mathcal{U}_{i+1, j}^{n}, \boldsymbol{e}_{1}\right)-\widehat{\mathbb{F}}\left(\mathcal{U}_{i-1, j}^{n}, \mathcal{U}_{i, j}^{n}, \boldsymbol{e}_{1}\right)\right) \\
+ & 2 \Delta t \widehat{\mathbb{S}}\left(\mathcal{U}_{i, j}^{n}, \nabla W, \boldsymbol{e}_{1}\right) .
\end{aligned}
$$

Next, let us introduce the state,

$$
\begin{aligned}
\mathcal{U}_{i, j}^{y}=\mathcal{U}_{i, j}^{n} & -\frac{\Delta t}{\Delta y / 2}\left(\widehat{\mathbb{F}}\left(\mathcal{U}_{i, j}^{n}, \mathcal{U}_{i, j+1}^{n}, \boldsymbol{e}_{2}\right)-\widehat{\mathbb{F}}\left(\mathcal{U}_{i, j-1}^{n}, \mathcal{U}_{i, j}^{n}, \boldsymbol{e}_{2}\right)\right) \\
+ & 2 \Delta t \widehat{\mathbb{S}}\left(\mathcal{U}_{i, j}^{n}, \nabla W, \boldsymbol{e}_{2}\right) .
\end{aligned}
$$

We easily deduce the following identity:

$$
\mathcal{U}_{i, j}^{n+1}=\frac{1}{2}\left(\mathcal{U}_{i, j}^{x}+\mathcal{U}_{i, j}^{y}\right)
$$

to now establish the expected robustness and stability properties.

Concerning the robustness, since $\Omega$ is a convex set, it suffices to prove that both $\mathcal{U}_{i, j}^{x}$ and $\mathcal{U}_{i, j}^{y}$ belong to $\Omega$. By robustness of the $1 \mathrm{D}$ scheme, under the half-CFL condition, we immediately have $\mathcal{U}_{i, j}^{x}$ in $\Omega$.

Concerning $\mathcal{U}_{i, j}^{y}$, by applying the rotational operator to the state $\mathcal{U}_{i, j}^{y}$, involving the rotational invariance of the numerical flux function and source term function (3.7), we get,

$$
\begin{aligned}
\bar{R}\left(\mathcal{U}_{i, j}^{y}\right)=\bar{R}\left(\mathcal{U}_{i, j}^{n}\right)- & \frac{\Delta t}{\Delta y / 2}\left(\widehat{\mathbb{F}}\left(\bar{R}\left(\mathcal{U}_{i, j}^{n}\right), \bar{R}\left(\mathcal{U}_{i, j+1}^{n}\right), \boldsymbol{e}_{1}\right)-\widehat{\mathbb{F}}\left(\bar{R}\left(\mathcal{U}_{i, j-1}^{n}\right), \bar{R}\left(\mathcal{U}_{i, j}^{n}\right), \boldsymbol{e}_{1}\right)\right) \\
& +2 \Delta t \widehat{\mathbb{S}}\left(\bar{R}\left(\mathcal{U}_{i, j}^{n}\right), R \nabla W, \boldsymbol{e}_{1}\right)
\end{aligned}
$$

as soon as $\boldsymbol{R e}_{2}=\boldsymbol{e}_{1}$. Since the states $\bar{R}\left(\mathcal{U}_{i, j}^{n}\right), \bar{R}\left(\mathcal{U}_{i, j \pm 1}^{n}\right)$ belong to $\Omega$, by arguing the robustness of the $1 \mathrm{D}$ scheme, we deduce $\bar{R}\left(\mathcal{U}_{i, j}^{y}\right) \in \Omega$. Because $\bar{R}(\Omega)=\Omega$, we obtain $\mathcal{U}_{i, j}^{y} \in \Omega$.

As a consequence, we have proved that $\mathcal{U}_{i, j}^{n+1}$ stays in $\Omega$.

Regarding discrete entropy inequalities, once again we argue the reformulation (3.11). According to the stability property (3.3), we have

$$
\rho F\left(s\left(\mathcal{U}_{i, j}^{x}\right)\right) \leq \rho F\left(s\left(\mathcal{U}_{i, j}^{n}\right)\right)-\frac{\Delta t}{\Delta x / 2}\left(\mathbb{G}\left(\mathcal{U}_{i, j}^{n}, \mathcal{U}_{i, j+1}^{n}, \boldsymbol{e}_{1}\right)-\mathbb{G}\left(\mathcal{U}_{i, j-1}^{n}, \mathcal{U}_{i, j}^{n}, \boldsymbol{e}_{1}\right)\right),
$$


and

$$
\begin{aligned}
\rho F\left(s\left(\bar{R}\left(\mathcal{U}_{i, j}^{y}\right)\right)\right) & \leq \rho F\left(s\left(\bar{R}\left(\mathcal{U}_{i, j}^{n}\right)\right)\right) \\
& -\frac{\Delta t}{\Delta y / 2}\left(\widehat{\mathbb{G}}\left(\bar{R}\left(\mathcal{U}_{i, j}^{n}\right), \bar{R}\left(\mathcal{U}_{i, j+1}^{n}\right), \boldsymbol{e}_{1}\right)-\widehat{\mathbb{G}}\left(\bar{R}\left(\mathcal{U}_{i, j-1}^{n}\right), \bar{R}\left(\mathcal{U}_{i, j}^{n}\right), \boldsymbol{e}_{1}\right)\right) .
\end{aligned}
$$

Invoking the assumption (3.8), with $R \boldsymbol{e}_{2}=\boldsymbol{e}_{1}$, the second relation rewrites

$$
\rho F\left(s\left(\mathcal{U}_{i, j}^{y}\right)\right) \leq \rho F\left(s\left(\mathcal{U}_{i, j}^{n}\right)\right)-\frac{\Delta t}{\Delta y / 2}\left(\widehat{\mathbb{G}}\left(\mathcal{U}_{i, j}^{n}, \mathcal{U}_{i, j+1}^{n}, \boldsymbol{e}_{2}\right)-\widehat{\mathbb{G}}\left(\mathcal{U}_{i, j-1}^{n}, \mathcal{U}_{i, j}^{n}, \boldsymbol{e}_{2}\right)\right)
$$

By taking the half-sum of the first inequality of (3.12) and (3.13), the discrete entropy inequality (3.9) is recovered.

Concerning the minimum entropy principle, from the definition of $\mathcal{U}_{i, j}^{x}$ and (3.4), we have

$$
s\left(\mathcal{U}_{i, j}^{x}\right) \geq \min \left(s_{i-1, j}^{n}, s_{i, j}^{n}, s_{i+1, j}^{n}\right),
$$

where we have set $s_{i, j}^{n}=s\left(\mathcal{U}_{i, j}^{n}\right)$ for all $(i, j) \in \mathbb{Z}^{2}$.

Similarly, by definition of $\bar{R}\left(\mathcal{U}_{i, j}^{y}\right)$, we have

$$
s\left(\bar{R}\left(\mathcal{U}_{i, j}^{y}\right)\right) \geq \min \left(s\left(\bar{R}\left(\mathcal{U}_{i, j-1}^{n}\right)\right), s\left(\bar{R}\left(\mathcal{U}_{i, j}^{n}\right)\right), s\left(\bar{R}\left(\mathcal{U}_{i, j+1}^{n}\right)\right)\right) .
$$

By invariance of $s$ under the rotational operator $\bar{R},(2.18)$, we get

$$
s\left(\mathcal{U}_{i, j}^{y}\right) \geq \min \left(s_{i, j-1}^{n}, s_{i, j}^{n}, s_{i, j+1}^{n}\right) .
$$

Since the function $\mathcal{U} \mapsto \rho F(s(\mathcal{U}))$ is convex, from (3.11) we have

$$
\rho_{i, j}^{n+1} F(s)_{i, j}^{n+1} \leq \frac{1}{2}(\rho F(s))\left(\mathcal{U}_{i, j}^{x}\right)+\frac{1}{2}(\rho F(s))\left(\mathcal{U}_{i, j}^{y}\right) .
$$

Because $F$ is a strictly decreasing function, we have

$$
\begin{aligned}
& F(s)\left(\mathcal{U}_{i, j}^{x}\right) \leq F\left(\min \left(s_{i-1, j}^{n}, s_{i, j-1}^{n}, s_{i, j}^{n}, s_{i+1, j}^{n}, s_{i, j+1}^{n}\right)\right), \\
& F(s)\left(\mathcal{U}_{i, j}^{y}\right) \leq F\left(\min \left(s_{i-1, j}^{n}, s_{i, j-1}^{n}, s_{i, j}^{n}, s_{i+1, j}^{n}, s_{i, j+1}^{n}\right)\right) .
\end{aligned}
$$

It follows that

$$
\rho_{i, j}^{n+1} F(s)_{i, j}^{n+1} \leq\left(\frac{1}{2} \rho_{i, j}^{x}+\frac{1}{2} \rho_{i, j}^{y}\right) F\left(\min \left(s_{i-1, j}^{n}, s_{i, j-1}^{n}, s_{i, j}^{n}, s_{i+1, j}^{n}, s_{i, j+1}^{n}\right)\right) .
$$

By noting that $\rho_{i, j}^{n+1}=\frac{1}{2} \rho_{i, j}^{x}+\frac{1}{2} \rho_{i, j}^{y}$ and since $F$ is a strictly decreasing function, the required minimum entropy principle (3.10) is obtained. The proof is thus complete.

Equipped with Theorem 3.1, we then consider the 1D model given by (3.2)

$$
\partial_{t} \mathcal{U}+\partial_{x} \mathcal{F}(\mathcal{U})=\mathcal{S}\left(\mathcal{U},\left(\boldsymbol{e}_{1} \otimes \boldsymbol{e}_{1}\right) \nabla W\right)
$$

where the vectors $\mathcal{U}, \mathcal{F}(\mathcal{U})$ and $\mathcal{S}\left(\mathcal{U},\left(\boldsymbol{e}_{1} \otimes \boldsymbol{e}_{1}\right) \boldsymbol{\nabla} W\right)$ are defined by (2.3)-(2.4). According to $(2.7)$, this $1 \mathrm{D}$ model can be rewritten as

$$
\partial_{t} \mathcal{U}+\partial_{x} \mathbb{F}\left(\mathcal{U}, e_{1}\right)=\mathbb{S}\left(\mathcal{U}, \nabla W, e_{1}\right)
$$




\section{A Relaxation Model}

The numerical approximation of the system (3.20) omitting the source term $\mathcal{S}\left(\mathcal{U},\left(\boldsymbol{e}_{1} \otimes \boldsymbol{e}_{1}\right) \boldsymbol{\nabla} W\right)$ has already been studied in [18, 5, 31] by Godunov-type schemes. In the present work, we design a Godunov-type scheme to approximate the system (2.2)-(2.5) which incorporates efficiently the source term $\mathcal{S}\left(\mathcal{U},\left(\boldsymbol{e}_{1} \otimes \boldsymbol{e}_{1}\right) \boldsymbol{\nabla} W\right)$. Following works on shallow water systems with gravity forces $[14,36]$ and astrophysical fluids with gravitational forces [11], we first propose to introduce the potential $\varphi^{e q}$ defined by,

$$
\varphi^{e q}(x, t)=\frac{1}{2} \int_{0}^{x} \rho(\xi, t) \partial_{\xi} W(\xi, t) d \xi
$$

The system (3.20) then rewrites

$$
\left\{\begin{array}{cccc}
\partial_{t} \rho & + & \partial_{x}\left(\rho u_{1}\right) & =0 \\
\partial_{t}\left(\rho u_{1}\right) & + & \partial_{x}\left(\rho u_{1}^{2}+\rho \varepsilon_{11}+\varphi^{e q}\right) & =0 \\
\partial_{t}\left(\rho u_{2}\right) & + & \partial_{x}\left(\rho u_{1} u_{2}+\rho \varepsilon_{12}\right) & =0 \\
\partial_{t}\left(\rho u_{1}^{2}+\rho \varepsilon_{11}\right) & + & \partial_{x}\left(\left(\rho u_{1}^{2}+\rho \varepsilon_{11}\right) u_{1}+2 \rho \varepsilon_{11} u_{1}\right)+2 u_{1} \partial_{x} \varphi^{e q} & =0 \\
\partial_{t}\left(\rho u_{1} u_{2}+\rho \varepsilon_{12}\right) & +\partial_{x}\left(\left(\rho u_{1} u_{2}+\rho \varepsilon_{12}\right) u_{1}+\rho \varepsilon_{12} u_{1}+\rho \varepsilon_{11} u_{2}\right)+u_{2} \partial_{x} \varphi^{e q} & =0 \\
\partial_{t}\left(\rho u_{2}^{2}+\rho \varepsilon_{22}\right) & + & \partial_{x}\left(\left(\rho u_{2}^{2}+\rho \varepsilon_{22}\right) u_{1}+2 \rho \varepsilon_{12} u_{2}\right) & =0 \\
\partial_{t}\left(\rho \varepsilon_{33}\right) & + & \partial_{x}\left(\rho \varepsilon_{33} u_{1}\right) & =0
\end{array}\right.
$$

We suggest to approximate weak solutions of (4.2) by weak solutions of a relaxation model which is a first order singular perturbation of (4.2). The reader is referred to $[28,15]$ for the pioneering works on relaxation models. Here, we adopt the relaxation model introduced in [5] (see [2], [13], [16], [22]), where most of nonlinearities of the initial model are relaxed. According to [5], the following Suliciu relaxation-type model is introduced,

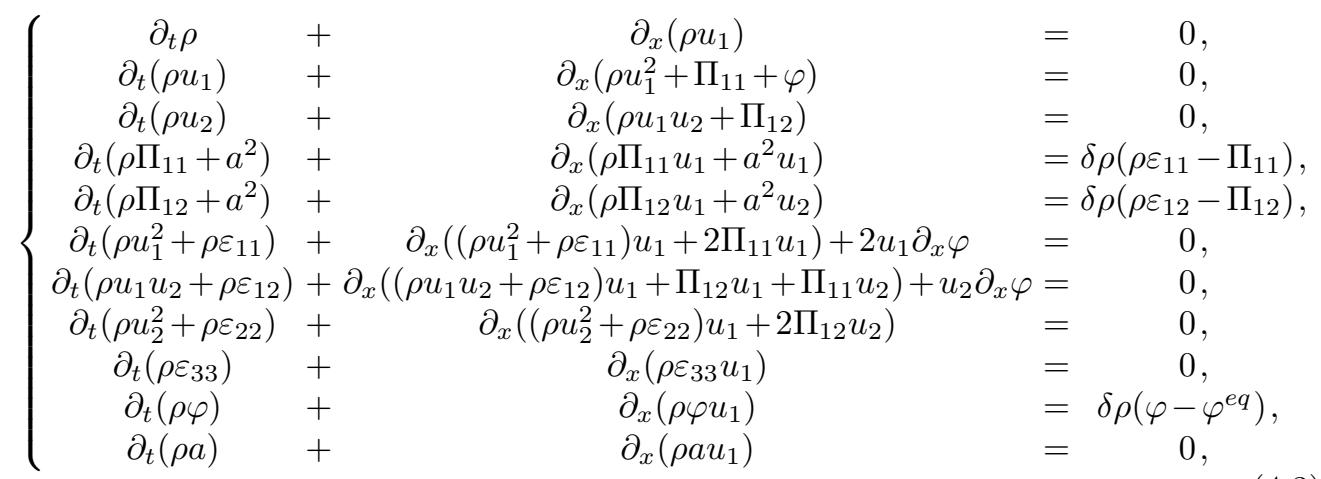

where the pressures $\Pi_{11}$, and $\Pi_{12}$ are the approximations of $\rho \varepsilon_{11}$, and $\rho \varepsilon_{12}$, respectively. Indeed, as soon as the relaxation parameter $\delta$ goes to infinity, the pressures $\rho \varepsilon_{11}$ and $\rho \varepsilon_{12}$ are recovered $[5,32]$. Simultaneously, the approximated potential $\varphi$ is relaxed to the expected value potential $\varphi^{e q}$. The evolution of the parameter $a$ is governed by the transport equation as proposed in [9]. This modification allows to chose different values of $a$ that accurately bound the Riemann problem wave velocities coming from the Suliciu relaxation model (4.3). We refer to [6] where another procedure to introduce different values of $a$ is proposed. The speed $a$ satisfies to some constraints, the so-called Whitham subcharacteristic condition which will be discussed later on. First, let us investigate the 
algebraic properties of (4.3). We introduce a vector $\mathbb{U}$ defined as follows,

$$
\mathbb{U}=\left(\begin{array}{c}
\rho \\
\rho u_{1} \\
\rho u_{2} \\
\rho \Pi_{11} \\
\rho \Pi_{12} \\
\rho u_{1}^{2}+\rho \varepsilon_{11} \\
\rho u_{1} u_{2}+\rho \varepsilon_{12} \\
\rho u_{2}^{2}+\rho \varepsilon_{22} \\
\rho \varepsilon_{33} \\
\rho \varphi \\
\rho a
\end{array}\right)
$$

belonging to the space

$$
\mathcal{V}=\left\{\mathbb{U} \in \mathbb{R}^{11} ; \rho>0\right\}
$$

The model (4.3) then rewrites

$$
\partial_{t} \mathbb{U}+\partial_{x} \overline{\mathbb{F}}(\mathbb{U})+\overline{\mathbb{A}}(\mathbb{U}) \partial_{x} \mathbb{U}=\delta \mathbb{T}(\mathbb{U}),
$$

where we have introduced:

$$
\overline{\mathbb{F}}(\mathbb{U})=\left(\begin{array}{c}
\rho u_{1} \\
\rho u_{1}^{2}+\Pi_{11}+\varphi \\
\rho u_{1} u_{2}+\Pi_{12} \\
\rho \Pi_{11} u_{1}+a^{2} u_{1} \\
\rho \Pi_{12} u_{1}+a^{2} u_{2} \\
\left(\rho u_{1}^{2}+\rho \varepsilon_{11}+2 \Pi_{11}\right) u_{1} \\
\rho u_{1}^{2} u_{2}+\rho \varepsilon_{12} u_{1}+\Pi_{12} u_{1}+\Pi_{11} u_{2} \\
\left(\rho u_{2}^{2}+\rho \varepsilon_{22}\right) u_{1}+2 \Pi_{12} u_{2} \\
\rho \varepsilon_{33} u_{1} \\
\rho \varphi u_{1} \\
\rho a u_{1}
\end{array}\right), \quad \mathbb{T}(\mathbb{U})=\left(\begin{array}{c}
0 \\
0 \\
0 \\
\rho\left(\rho \varepsilon_{11}-\Pi_{11}\right) \\
\rho\left(\rho \varepsilon_{12}-\Pi_{12}\right) \\
0 \\
0 \\
0 \\
0 \\
\rho\left(\varphi-\varphi^{e q}\right) \\
0
\end{array}\right)
$$

and $\overline{\mathbb{A}}(\mathbb{U})$ defined by

$$
\overline{\mathbb{A}}(\mathbb{U}) \partial_{x} \mathbb{U}=\left(\begin{array}{lllllllllll}
0 & 0 & 0 & 0 & 0 & 2 u_{1} \partial_{x} \varphi & u_{2} \partial_{x} \varphi & 0 & 0 & 0 & 0
\end{array}\right)^{T} .
$$

Now, let us recall the close relationships between the relaxation system (4.3) and the initial model (4.2). Indeed, there exists a projection matrix, denoted by $\mathcal{N}$, with rank 7 which satisfies

$$
\mathcal{N} \mathbb{T}(\mathbb{U})=0 \quad \text { and } \quad \mathcal{N}(\mathbb{U})=\mathcal{U}
$$

Of course, this matrix is obviously given by

$$
\mathcal{N}=\left(\begin{array}{lllllllllll}
1 & 0 & 0 & 0 & 0 & 0 & 0 & 0 & 0 & 0 & 0 \\
0 & 1 & 0 & 0 & 0 & 0 & 0 & 0 & 0 & 0 & 0 \\
0 & 0 & 1 & 0 & 0 & 0 & 0 & 0 & 0 & 0 & 0 \\
0 & 0 & 0 & 0 & 0 & 1 & 0 & 0 & 0 & 0 & 0 \\
0 & 0 & 0 & 0 & 0 & 0 & 1 & 0 & 0 & 0 & 0 \\
0 & 0 & 0 & 0 & 0 & 0 & 0 & 1 & 0 & 0 & 0 \\
0 & 0 & 0 & 0 & 0 & 0 & 0 & 0 & 1 & 0 & 0
\end{array}\right) .
$$


Moreover, by definition of $\Omega$ and $\mathcal{V}$, we have

$$
\mathcal{N} \mathcal{\supsetneq} \Omega
$$

In addition, the source term $\mathbb{T}: \mathbb{R}^{11} \rightarrow \mathbb{R}^{11}$ admits equilibrium solutions $\mathbb{U}^{e q}$ such that $\mathbb{T}\left(\mathbb{U}^{e q}\right)=0$. These equilibrium states, the so-called Maxwellians, are parametrized as follows:

$$
\mathbb{U}^{e q}:=\mathbb{U}^{e q}\left(\mathcal{U} ; \varphi^{e q}, a\right)
$$

As a consequence, we have

$$
\mathcal{N} \mathbb{U}^{e q}\left(\mathcal{U} ; \varphi^{e q}, a\right)=\mathcal{U}
$$

According to the derivation of the relaxation model, the main interest coming from (4.3) stays in a suitable linearization making the associated Riemann problem easily solvable. Here, such a property is deduced from the linearly degenerated property satisfied by all the fields, as given in the following result.

Lemma 4.1. Assume $a>0$ and $\delta=0$. For all $\mathbb{U} \in \mathcal{V}$ the system (4.3) is hyperbolic and its eigenvalues are given by

$$
\left\{\begin{array}{l}
\mu_{1}(\mathbb{U})=\mu_{2}(\mathbb{U})=u_{1}-\frac{a}{\rho} \\
\mu_{3}(\mathbb{U})=\mu_{4}(\mathbb{U})=\mu_{5}(\mathbb{U})=\mu_{6}(\mathbb{U})=\mu_{7}(\mathbb{U})=\mu_{8}(\mathbb{U})=\mu_{9}(\mathbb{U})=u_{1}, \\
\mu_{10}(\mathbb{U})=\mu_{11}(\mathbb{U})=u_{1}+\frac{a}{\rho}
\end{array}\right.
$$

Moreover, all the associated characteristic fields are linearly degenerated.

Proof. First, let us exhibit the algebra coming from the homogeneous system associated to (4.3), i.e. we enforce $\delta=0$. By involving primitive variables, the system writes as follows

$$
\left\{\begin{array}{ccc}
\partial_{t} \rho+ & u_{1} \partial_{x} \rho+\rho \partial_{x} u_{1} & =0 \\
\partial_{t} u_{1}+ & u_{1} \partial_{x} u_{1}+\frac{1}{\rho} \partial_{x} \Pi_{11}+\frac{1}{\rho} \partial_{x} \varphi & =0 \\
\partial_{t} u_{2}+ & u_{1} \partial_{x} u_{2}+\frac{1}{\rho} \partial_{x} \Pi_{12} & =0 \\
\partial_{t} \Pi_{11}+u_{1} \partial_{x} \Pi_{11}+\frac{a^{2}}{\rho} \partial_{x} u_{1}+\frac{2 a u_{1}}{\rho} \partial_{x} a & =0 \\
\partial_{t} \Pi_{12}+ & u_{1} \partial_{x} \Pi_{12}+\frac{a^{2}}{\rho} \partial_{x} u_{2}+\frac{2 a u_{2}}{\rho} \partial_{x} a & =0 \\
\partial_{t} \varepsilon_{11}+ & u_{1} \partial_{x} \varepsilon_{11}+2 \frac{\Pi_{11}}{\rho} \partial_{x} u_{1} & =0 \\
\partial_{t} \varepsilon_{12}+u_{1} \partial_{x} \varepsilon_{12}+\frac{\Pi_{12}}{\rho} \partial_{x} u_{1}+\frac{\Pi_{11}}{\rho} \partial_{x} u_{2} & =0 \\
\partial_{t} \varepsilon_{22}+ & u_{1} \partial_{x} \varepsilon_{22}+2 \frac{\Pi_{12}}{\rho} \partial_{x} u_{2} & =0 \\
\partial_{t} \varepsilon_{33}+ & u_{1} \partial_{x} \varepsilon_{33} & =0 \\
\partial_{t} \varphi+ & u_{1} \partial_{x} \varphi & =0 \\
\partial_{t} a+ & u_{1} \partial_{x} a & =0 .
\end{array}\right.
$$

We have

$$
\partial_{t} \mathbb{V}+\overline{\mathcal{A}}(\mathbb{V}) \partial_{x} \mathbb{V}=0
$$


where

$$
\mathbb{V}=\left(\begin{array}{c}
\rho \\
u_{1} \\
u_{2} \\
\Pi_{11} \\
\Pi_{12} \\
\varepsilon_{11} \\
\varepsilon_{12} \\
\varepsilon_{22} \\
\varepsilon_{33} \\
\varphi \\
a
\end{array}\right), \overline{\mathcal{A}}(\mathbb{V})=\left(\begin{array}{ccccccccccc}
u_{1} & \rho & 0 & 0 & 0 & 0 & 0 & 0 & 0 & 0 & 0 \\
0 & u_{1} & 0 & 1 / \rho & 0 & 0 & 0 & 0 & 0 & 1 / \rho & 0 \\
0 & 0 & u_{1} & 0 & 1 / \rho & 0 & 0 & 0 & 0 & 0 & 0 \\
0 & a^{2} / \rho & 0 & u_{1} & 0 & 0 & 0 & 0 & 0 & 0 & 2 a u_{1} / \rho \\
0 & 0 & a^{2} / \rho & 0 & u_{1} & 0 & 0 & 0 & 0 & 0 & 2 a u_{2} / \rho \\
0 & 2 \Pi_{11} / \rho & 0 & 0 & 0 & u_{1} & 0 & 0 & 0 & 0 & 2 u_{1} \\
0 & \Pi_{12} / \rho & \Pi_{11} / \rho & 0 & 0 & 0 & u_{1} & 0 & 0 & 0 & u_{2} \\
0 & 0 & 2 \Pi_{12} / \rho & 0 & 0 & 0 & 0 & u_{1} & 0 & 0 & 0 \\
0 & 0 & 0 & 0 & 0 & 0 & 0 & 0 & u_{1} & 0 & 0 \\
0 & 0 & 0 & 0 & 0 & 0 & 0 & 0 & 0 & u_{1} & 0 \\
0 & 0 & 0 & 0 & 0 & 0 & 0 & 0 & 0 & 0 & u_{1}
\end{array}\right)
$$

Straightforward computations show that $u_{1}$ is an eigenvalue of $\overline{\mathcal{A}}(\mathbb{V})$ of multiplicity 7 , while $u_{1} \pm a / \rho$ are eigenvalues of $\overline{\mathcal{A}}(\mathbb{V})$ of multiplicity 2 . In addition, each characteristic field is linearly degenerated.

Next, we are concerned with solving the Riemann problem associated to the relaxation model (4.3) with $\delta=0$. Since all characterics fields are linearly degenerated, the exact Riemann solution consists of four constant states separated by three contact discontinuities described in the following result.

Lemma 4.2. Assume $\mathbb{U}_{l}$ and $\mathbb{U}_{r}$ are constant states in $\mathcal{V}$ and consider

$$
\mathbb{U}_{0}(x)= \begin{cases}\mathbb{U}_{l} & \text { if } x<0 \\ \mathbb{U}_{r} & \text { if } x>0\end{cases}
$$

as the initial data for the system (4.3) with $\delta=0$. Let $a_{l}>0, a_{r}>0$ and assume the following condition is satisfied

$$
b_{l}=\mu_{1}\left(\mathbb{U}_{l}\right)=u_{1, l}-\frac{a_{l}}{\rho_{l}}<u_{1}^{\star}<\mu_{11}\left(\mathbb{U}_{r}\right)=u_{1, r}+\frac{a_{r}}{\rho_{r}}=b_{r},
$$

where

$$
u_{1}^{\star}=\frac{a_{l} u_{1, l}+a_{r} u_{1, r}}{a_{l}+a_{r}}-\frac{\Pi_{11, r}-\Pi_{11, l}}{a_{l}+a_{r}}-\frac{\varphi_{r}-\varphi_{l}}{a_{l}+a_{r}} .
$$

Then the weak solution of system (4.3) with $\delta=0$ supplemented by the initial data defined by (4.15) is given by

$$
\mathbb{U}_{\mathcal{R}}\left(\frac{x}{t}, \mathbb{U}_{l}, \mathbb{U}_{r}\right)=\left\{\begin{array}{lll}
\mathbb{U}_{l}, & \text { if } & b_{l}>\frac{x}{t}, \\
\mathbb{U}_{l}^{\star}, & \text { if } & b_{l} \leq \frac{x}{t} \leq u_{1}^{\star}, \\
\mathbb{U}_{r}^{\star}, & \text { if } & u_{1}^{\star} \leq \frac{x}{t} \leq b_{r}, \\
\mathbb{U}_{r}, & \text { if } & b_{r}<\frac{x}{t}
\end{array}\right.
$$


With $g=l, r$, let us introduce the following notations,

$$
\left\{\begin{array}{l}
u_{1}^{\star}=\frac{a_{l} u_{1, l}+a_{r} u_{1, r}}{a_{l}+a_{r}}-\frac{\Pi_{11, r}-\Pi_{11, l}}{a_{l}+a_{r}}-\frac{\varphi_{r}-\varphi_{l}}{a_{l}+a_{r}}, \\
u_{2}^{\star}=\frac{a_{l} u_{2, l}+a_{r} u_{2, r}}{a_{r}+a_{l}}-\frac{\Pi_{12, r}-\Pi_{12, l}}{a_{r}+a_{l}}, \\
\Pi_{11, l}^{\star}=\Pi_{11, l}-a_{l}\left(u_{1}^{\star}-u_{1, l}\right), \\
\Pi_{11, r}^{\star}=\Pi_{11, l}^{\star}-\left(\varphi_{r}-\varphi_{l}\right), \\
\Pi_{12}^{\star}=\Pi_{12, r}+a_{r}\left(u_{2}^{\star}-u_{2, r}\right)=\Pi_{12, l}-a_{l}\left(u_{2}^{\star}-u_{2, l}\right), \\
\frac{1}{\rho_{g}^{\star}}=\frac{1}{\rho_{g}}-\frac{\Pi_{11, g}^{\star}-\Pi_{11, g}}{\left(a_{g}\right)^{2}}, \\
\varepsilon_{11, g}^{\star}=\varepsilon_{11, g}+\frac{\left(\Pi_{11, g}^{\star}\right)^{2}-\left(\Pi_{11, g}\right)^{2}}{\left(a_{g}\right)^{2}}, \\
\varepsilon_{12, g}^{\star}=\varepsilon_{12, g}+\frac{\Pi_{11, g}^{\star} \Pi_{12}^{\star}-\Pi_{11, g} \Pi_{12, g}}{\left(a_{g}\right)^{2}} \\
\varepsilon_{22, g}^{\star}=\varepsilon_{22, g}+\frac{\left(\Pi_{12}^{\star}\right)^{2}-\left(\Pi_{12, g}\right)^{2}}{\left(a_{g}\right)^{2}}, \\
\varepsilon_{33, g}^{\star}=\varepsilon_{33, g}, \\
\varphi_{g}^{\star}=\varphi_{g}, \\
a_{g}^{\star}=a_{g} .
\end{array}\right.
$$

Then the star intermediate states $\mathbb{U}_{l}^{\star}$ and $\mathbb{U}_{r}^{\star}$ in $\mathcal{V}$ are given by

$$
\mathbb{U}_{g}^{\star}=\left(\begin{array}{c}
\rho_{g}^{\star} \\
\rho_{g}^{\star} u_{1}^{\star} \\
\rho_{g}^{\star} u_{2}^{\star} \\
\rho_{g}^{\star} \Pi_{11, g}^{\star}+a_{g}^{\star} \\
\rho_{g}^{\star} \Pi_{12}^{\star}+a_{g}^{\star} \\
\rho_{g}^{\star}\left(u_{1}^{\star}\right)^{2}+\rho_{g}^{\star} \varepsilon_{11, g}^{\star} \\
\rho_{g}^{\star} u_{1}^{\star} u_{2}^{\star}+\rho_{g}^{\star} \varepsilon_{12, g}^{\star} \\
\rho_{g}^{\star}\left(u_{2}^{\star}\right)^{2}+\rho_{g}^{\star} \varepsilon_{22, g}^{\star} \\
\rho_{g}^{\star} \varepsilon_{33, g}^{\star} \\
\rho_{g}^{\star} \varphi_{g}^{\star} \\
\rho_{g}^{\star} a_{g}^{\star}
\end{array}\right) .
$$

Proof. After straightfoward computations (see also [20]), the Riemann invariants associated to the eigenvalue $u_{1}$ are

$$
u_{1}, u_{2}, \Pi_{12}, \Pi_{11}+\varphi
$$

while those coming from the eigenvalue $u_{1}-a / \rho$ are

$$
\begin{aligned}
& a, \varphi, \varepsilon_{33}, \varepsilon_{12}-\Pi_{11} \Pi_{12} / a^{2}, \varepsilon_{11}-\left(\Pi_{11}\right)^{2} / a^{2}, \varepsilon_{22}-\left(\Pi_{12}\right)^{2} / a^{2}, \Pi_{11}+a^{2} / \rho, \\
& \Pi_{11}+a u_{1}, \Pi_{12}+a u_{2} .
\end{aligned}
$$

Finally, the Riemann invariants associated to the eigenvalue $u_{1}+a / \rho$ are

$$
\begin{aligned}
& a, \varphi, \varepsilon_{33}, \varepsilon_{12}-\Pi_{11} \Pi_{12} / a^{2}, \varepsilon_{11}-\left(\Pi_{11}\right)^{2} / a^{2}, \varepsilon_{22}-\left(\Pi_{12}\right)^{2} / a^{2}, \Pi_{11}+a^{2} / \rho, \\
& \Pi_{11}-a u_{1}, \Pi_{12}-a u_{2} .
\end{aligned}
$$


Involving the continuity of the Riemann invariants across their associated linearly fields immediately imposes the intermediate states $\mathbb{U}_{l}^{\star}$ and $\mathbb{U}_{r}^{\star}$ to be defined by $(4.19)-(4.20)$. The proof is thus achieved.

Let us emphasize that the hypothesis (4.16) is essential and it is always satisfied for a reasonable choice of positive real numbers $a_{l}$, and $a_{r}$. In fact, we have the following result.

LEMmA 4.3. For all $\mathbb{U}_{l}$ and $\mathbb{U}_{r}$ given in $\mathcal{V}$ with $\mathcal{N} \mathbb{U}_{l}, \mathcal{N} \mathbb{U}_{r} \in \Omega$, there exist two real numbers $a_{l}>0$, and $a_{r}>0$ with $\frac{a_{l}}{a_{r}}=O(1)$ such that the intermediate states $\mathbb{U}_{l}^{\star}$ and $\mathbb{U}_{r}^{\star}$, defined in Lemma 4.2, belong to $\mathcal{V}$, and $\mathcal{N} \mathbb{U}_{l}^{\star}, \mathcal{N} \mathbb{U}_{r}^{\star} \in \Omega$.

By involving the Riemann invariants, we easily notice the equivalence between the estimates (4.16) and the positiveness of the intermediate densities $\rho_{l, r}^{\star}$.

Proof. For the sake of simplicity, we set $\mathrm{J} \alpha \mathrm{K}=\alpha_{r}-\alpha_{l}$. Then from (4.19), we deduce

$$
\left\{\begin{array}{l}
\frac{1}{\rho_{l}^{\star}}=\frac{1}{\rho_{l}}+\frac{a_{r} \mathrm{~J} u_{1} \mathrm{~K}-\mathrm{J}_{11} \mathrm{~K}-\mathrm{J} \varphi \mathrm{K}}{a_{l}\left(a_{l}+a_{r}\right)}, \\
\frac{1}{\rho_{r}^{\star}}=\frac{1}{\rho_{r}}+\frac{a_{l} \mathrm{~J} u_{1} \mathrm{~K}+\mathrm{J} \Pi_{11} \mathrm{~K}+\mathrm{J} \varphi \mathrm{K}}{a_{r}\left(a_{l}+a_{r}\right)} .
\end{array}\right.
$$

Since $\rho_{l}>0, \rho_{r}>0, a_{l}>0, a_{r}>0$, the positiveness of $\rho_{l}^{\star}$ and $\rho_{r}^{\star}$ resulting from (4.24) turns into

$$
\left\{\begin{array}{l}
a_{l}\left(a_{l}+a_{r}\right)+\rho_{l}\left(a_{r} \mathrm{~J} u_{1} \mathrm{~K}-\mathrm{J} \Pi_{11} \mathrm{~K}-\mathrm{J} \varphi \mathrm{K}\right)>0 \\
a_{r}\left(a_{l}+a_{r}\right)+\rho_{r}\left(a_{l} \mathrm{~J} u_{1} \mathrm{~K}+\mathrm{J} \Pi_{11} \mathrm{~K}+\mathrm{J} \varphi \mathrm{K}\right)>0 .
\end{array}\right.
$$

We clearly obtain the required positiveness as soon as $a_{l}$ and $a_{r}$ are large enough. Nevertheless, in the specific case $a_{l}=a_{r}=a$, we can easily determine the minimum value of the unique parameter $a$ such that the intermediate densities are positive. Indeed, the relation (4.25) writes

$$
\left\{\begin{array}{l}
2 a^{2}+\rho_{l} \mathrm{~J} u_{1} \mathrm{~K} a-\rho_{l}\left(\mathrm{~J}_{11} \mathrm{~K}+\mathrm{J} \varphi \mathrm{K}\right)>0, \\
2 a^{2}+\rho_{r} \mathrm{~J} u_{1} \mathrm{~K} a+\rho_{r}\left(\mathrm{~J} \Pi_{11} \mathrm{~K}+\mathrm{J} \varphi \mathrm{K}\right)>0 .
\end{array}\right.
$$

We introduce

$$
\left\{\begin{array}{l}
a_{I}^{ \pm}=\frac{-\rho_{l} \mathrm{~J} u_{1} \mathrm{~K} \pm \sqrt{\Delta_{I}}}{4} \quad \text { whenever } \quad \Delta_{I}>0, \\
a_{I I}^{ \pm}=\frac{-\rho_{r} \mathrm{~J} u_{1} \mathrm{~K} \pm \sqrt{\Delta_{I I}}}{4} \quad \text { whenever } \quad \Delta_{I I}>0,
\end{array}\right.
$$

where we have set

$$
\left\{\begin{array}{l}
\Delta_{I}=\left(\rho_{l} \mathrm{~J} u_{1} \mathrm{~K}\right)^{2}+8 \rho_{l}\left(\mathrm{~J} \Pi_{11} \mathrm{~K}+\mathrm{J} \varphi \mathrm{K}\right), \\
\Delta_{I I}=\left(\rho_{r} \mathrm{~J} u_{1} \mathrm{~K}\right)^{2}-8 \rho_{r}\left(\mathrm{~J} \Pi_{11} \mathrm{~K}+\mathrm{J} \varphi \mathrm{K}\right),
\end{array}\right.
$$

to get the following alternatives:

$$
\left\{\begin{array}{l}
\bullet \Delta_{I}<0 \text { and } \Delta_{I I}<0 \text { then any } a>0, \\
\bullet \Delta_{I}<0 \text { and } \Delta_{I I}>0 \text { then } a>\max \left(\left|a_{I I}^{ \pm}\right|\right), \\
\bullet \Delta_{I}>0 \text { and } \Delta_{I I}<0 \text { then } a>\max \left(\left|a_{I}^{ \pm}\right|\right), \\
\bullet \Delta_{I}>0 \text { and } \Delta_{I I}>0 \text { then } a>\max \left(\left|a_{I}^{ \pm}\right|,\left|a_{I I}^{ \pm}\right|\right),
\end{array}\right.
$$


to satisfy $(4.26)$.

We now focus on the positiveness of the intermediate internal energy $\varepsilon_{l}^{\star}$, that of $\varepsilon_{r}^{\star}$ is similar. To simplify the notations, let us introduce

$$
\left\{\begin{array}{l}
\mathcal{X}=u_{1}^{\star}-u_{1, l}=\frac{a_{r} \mathrm{~J} u_{1} \mathrm{~K}-\mathrm{J} \Pi_{11} \mathrm{~K}-\mathrm{J} \varphi \mathrm{K}}{a_{l}+a_{r}}, \\
\mathcal{Y}=u_{2}^{\star}-u_{2, l}=\frac{a_{r} \mathrm{~J} u_{2} \mathrm{~K}-\mathrm{J} \Pi_{12} \mathrm{~K}}{a_{l}+a_{r}} .
\end{array}\right.
$$

The internal energies $\varepsilon_{11, l}^{\star}, \varepsilon_{12, l}^{\star}$ and $\varepsilon_{22, l}^{\star}$ defined by (4.19), write:

$$
\left\{\begin{array}{l}
\varepsilon_{11, l}^{\star}=\varepsilon_{11, l}-\left(\frac{\Pi_{11, l}}{a_{l}}\right)^{2}+\left(\mathcal{X}-\frac{\Pi_{11, l}}{a_{l}}\right)^{2}, \\
\varepsilon_{22, l}^{\star}=\varepsilon_{22, l}-\left(\frac{\Pi_{12, l}}{a_{l}}\right)^{2}+\left(\mathcal{Y}-\frac{\Pi_{12, l}}{a_{l}}\right)^{2}, \\
\varepsilon_{12, l}^{\star}=\varepsilon_{12, l}-\left(\frac{\Pi_{11, l}}{a_{l}}\right)\left(\frac{\Pi_{12, l}}{a_{l}}\right)+\left(\mathcal{X}-\frac{\Pi_{11, l}}{a_{l}}\right)\left(\mathcal{Y}-\frac{\Pi_{12, l}}{a_{l}}\right) .
\end{array}\right.
$$

As soon as $a_{l}$ fulfills

$$
a_{l}>\max \left(\frac{\left|\Pi_{11, l}\right|}{\sqrt{\varepsilon_{11, l}}}, \frac{\left|\Pi_{12, l}\right|}{\sqrt{\varepsilon_{22, l}}}\right),
$$

we get the required positiveness of the internal energies $\varepsilon_{11, l}^{\star}$ and $\varepsilon_{22, l}^{\star}$.

Finally, we study the positiveness of $\varepsilon_{11, l}^{\star} \varepsilon_{22, l}^{\star}-\left(\varepsilon_{12, l}^{\star}\right)^{2}$. By considering $\mathcal{X}$ and $\mathcal{Y}$, we have

$$
\begin{aligned}
\left(\varepsilon_{11, l}^{\star} \varepsilon_{22, l}^{\star}-\left(\varepsilon_{12, l}^{\star}\right)^{2}\right)=\left(\varepsilon_{22, l}\right. & \left.-\left(\frac{\Pi_{12, l}}{a_{l}}\right)^{2}\right) \mathcal{X}^{2} \\
+ & 2\left[\varepsilon_{12, l} \frac{\Pi_{12, l}}{a_{l}}-\varepsilon_{22, l} \frac{\Pi_{11, l}}{a_{l}}-\left(\varepsilon_{12, l}-\frac{\Pi_{11, l}}{a_{l}} \frac{\Pi_{12, l}}{a_{l}}\right) \mathcal{Y}\right] \mathcal{X} \\
& +\left[\varepsilon_{11, l}-\left(\frac{\Pi_{11, l}}{a_{l}}\right)^{2}\right] \mathcal{Y}^{2}+2\left[\varepsilon_{12, l} \frac{\Pi_{11, l}}{a_{l}}-\varepsilon_{11, l} \frac{\Pi_{12, l}}{a_{l}}\right] \mathcal{Y} \\
& +\varepsilon_{11, l} \varepsilon_{22, l}-\left(\varepsilon_{12, l}\right)^{2} .
\end{aligned}
$$

The discriminant of the above expression with respect to $\mathcal{X}$ is given by

$$
\Delta_{\mathcal{X}}=\frac{4 \mathcal{D}}{\left(a_{l}\right)^{2}}\left(\mathcal{Y}^{2}-2 \frac{\Pi_{12, l}}{a_{l}} \mathcal{Y}+\varepsilon_{22, l}\right) .
$$

where

$$
\mathcal{D}=\left(\varepsilon_{12, l}^{2}-\varepsilon_{11, l} \varepsilon_{22, l}\right)\left(a_{l}\right)^{2}+\varepsilon_{22, l}\left(\Pi_{11, l}\right)^{2}-2 \varepsilon_{12, l} \Pi_{12, l} \Pi_{11, l}+\varepsilon_{11}\left(\Pi_{12, l}\right)^{2} .
$$

The discriminant of $\Delta_{\mathcal{X}}$ with respect to $\mathcal{Y}$ writes

$$
\left(\Delta_{\mathcal{X}}\right)_{\mathcal{Y}}=-\frac{64 \mathcal{D}^{2}}{\left(a_{l}\right)^{6}}\left(\varepsilon_{22, l}\left(a_{l}\right)^{2}-\left(\Pi_{12, l}\right)^{2}\right) .
$$


According to $(4.32)$, we get $\left(\Delta_{\mathcal{X}}\right)_{\mathcal{Y}}<0$ and then $\Delta_{\mathcal{X}}<0$ for all $\mathcal{X}$ and $\mathcal{Y}$ whenever

$$
\mathcal{D}<0
$$

or equivalently

$$
\left(\varepsilon_{11, l} \varepsilon_{22, l}-\varepsilon_{12, l}^{2}\right)\left(a_{l}\right)^{2}-\left(\varepsilon_{22, l}\left(\Pi_{11, l}\right)^{2}-2 \varepsilon_{12, l} \Pi_{12, l} \Pi_{11, l}+\varepsilon_{11, l}\left(\Pi_{12, l}\right)^{2}\right)>0
$$

This is satisfied as soon as

$$
\left(a_{l}\right)^{2}>\left(\begin{array}{l}
\Pi_{11, l} \\
\Pi_{12, l}
\end{array}\right)^{T} \cdot \tilde{\varepsilon}^{-1} \cdot\left(\begin{array}{c}
\Pi_{11, l} \\
\Pi_{12, l}
\end{array}\right)
$$

where the positive definite matrix $\tilde{\varepsilon}$ is defined by

$$
\tilde{\varepsilon}=\left(\begin{array}{ll}
\varepsilon_{11, l} & \varepsilon_{12, l} \\
\varepsilon_{12, l} & \varepsilon_{22, l}
\end{array}\right) .
$$

As a consequence, $a_{l}$ can be chosen large enough to satisfy (4.37). The proof of the lemma is thus complete.

\section{A Relaxation Scheme}

Let us describe the finite volume scheme coming from this approximate Riemann solver and intended to approach the solution of the relaxation model (4.6). At time $t^{n}$, we consider a piecewise constant approximation of the solution of the initial model (4.2) given by,

$$
\mathcal{U}^{\Delta}\left(x, t^{n}\right)=\mathcal{U}_{i}^{n}, \quad x \in\left(x_{i-1 / 2}, x_{i+1 / 2}\right),
$$

where

$$
\mathcal{U}_{i}^{n}=\left(\begin{array}{c}
\rho_{i}^{n} \\
\rho_{i}^{n} u_{1, i}^{n} \\
\rho_{i}^{n} u_{2, i}^{n} \\
\rho_{i}^{n}\left(u_{1, i}^{n}\right)^{2}+P_{11, i}^{n} \\
\rho_{i}^{n} u_{1, i}^{n} u_{2, i}^{n}+P_{12, i}^{n} \\
\rho_{i}^{n}\left(u_{2, i}^{n}\right)^{2}+P_{22, i}^{n} \\
P_{33, i}^{n}
\end{array}\right)
$$

with $P_{11, i}^{n}=\rho_{i}^{n} \varepsilon_{11, i}^{n}, P_{12, i}^{n}=\rho_{i}^{n} \varepsilon_{12, i}^{n}, P_{22, i}^{n}=\rho_{i}^{n} \varepsilon_{22, i}^{n}, P_{33, i}^{n}=\rho_{i}^{n} \varepsilon_{33, i}^{n}$.

To evolve in time this approximation, we proceed in two steps:

First step : Evolution step. According to the equilibrium state definition, introduced in $(4.11)$, we set

$$
\mathbb{U}_{i}^{e q, n}:=\mathbb{U}^{e q}\left(\mathcal{U}_{i}^{n} ; \varphi_{i}^{e q, n}, a_{i}^{n}\right)
$$


Hence for all $i \in \mathbb{Z}$, we have

$$
\mathbb{U}_{i}^{e q, n}=\left(\begin{array}{c}
\rho_{i}^{n} \\
\rho_{i}^{n} u_{1, i}^{n} \\
\rho_{i}^{n} u_{2, i}^{n} \\
\rho_{i}^{n} P_{11, i}^{n}+a_{i}^{n} \\
\rho_{i}^{n} P_{12, i}^{n}+a_{i}^{n} \\
\rho_{i}^{n}\left(u_{1, i}^{n}\right)^{2}+P_{11, i}^{n} \\
\rho_{i}^{n} u_{1, i}^{n} u_{2, i}^{n}+P_{12, i}^{n} \\
\rho_{i}^{n}\left(u_{2, i}^{n}\right)^{2}+P_{22, i}^{n} \\
P_{33, i}^{n} \\
\rho_{i}^{n} \varphi_{i}^{e q, n} \\
\rho_{i}^{n} a_{i}^{n}
\end{array}\right)
$$

where the characterization of the positive real numbers $a_{i}^{n}$ satisfy Lemma 4.3. Additional conditions devoted to the choice of $a_{i}^{n}$ will be discussed later on.

At this level, the quantity $\varphi_{i}^{e q, n}$ is an approximation, at equilibrium, of the integral

$$
\frac{1}{2} \int_{0}^{x_{i}} \rho\left(\xi, t^{n}\right) \partial_{\xi} W\left(\xi, t^{n}\right) d \xi,
$$

to be defined later on.

To evolve in time this approximation, we consider the juxtaposition of the approximate Riemann problems solution $\mathbb{U}_{\mathcal{R}}\left(\frac{x-x_{i+1 / 2}}{t} ; \mathbb{U}_{i}^{e q, n}, \mathbb{U}_{i+1}^{e q, n}\right)$ stated at each interface $x_{i+1 / 2}$. As soon as $t$ belongs to $(0, \Delta t)$ with $\Delta t$ small enough such that

$$
\begin{aligned}
& \frac{\Delta t}{\Delta x} \max _{i \in \mathbb{Z}}\left(\left|b_{l, i+1 / 2}\right|,\left|b_{r, i+1 / 2}\right|\right) \leq \frac{1}{2}, \\
& b_{l, i+1 / 2}=u_{1, i}^{n}-\frac{a_{i}^{n}}{\rho_{i}^{n}}, \quad b_{r, i+1 / 2}=u_{1, i+1}^{n}+\frac{a_{i+1}^{n}}{\rho_{i+1}^{n}},
\end{aligned}
$$

the Riemann solvers do not interact. As a consequence, for all $t \in(0, \Delta t)$, we define

$$
\mathbb{U}^{\Delta}\left(x, t^{n}+t\right)=\mathbb{U}_{\mathcal{R}}\left(\frac{x-x_{i+1 / 2}}{t} ; \mathbb{U}_{i}^{e q, n}, \mathbb{U}_{i+1}^{e q, n}\right), \quad x \in\left(x_{i}, x_{i+1}\right) .
$$

From this solution a constant piecewise function is constructed by projection as follows,

$$
\overline{\mathbb{U}}_{i}^{n+1}=\frac{1}{\Delta x} \int_{x_{i-1 / 2}}^{x_{i+1 / 2}} \mathbb{U}^{\Delta}\left(x, t^{n}+\Delta t\right) d x .
$$

We introduce the following quantities

$$
\begin{aligned}
& \overline{\mathbb{U}}_{i+1 / 2}^{n+1,-}=\frac{1}{\Delta x} \int_{x_{i}}^{x_{i+1 / 2}} \mathbb{U}^{\Delta}\left(x, t^{n}+\Delta t\right) d x, \\
& \overline{\mathbb{U}}_{i+1 / 2}^{n+1,+}=\frac{1}{\Delta x} \int_{x_{i+1 / 2}}^{x_{i+1}} \mathbb{U}^{\Delta}\left(x, t^{n}+\Delta t\right) d x,
\end{aligned}
$$

such that the updated state (5.7) reads

$$
\overline{\mathbb{U}}_{i}^{n+1}=\overline{\mathbb{U}}_{i-1 / 2}^{n+1,+}+\overline{\mathbb{U}}_{i+1 / 2}^{n+1,-} .
$$


Practical expressions of the quantities $\overline{\mathbb{U}}_{i+1 / 2}^{n+1,+}$ and $\overline{\mathbb{U}}_{i+1 / 2}^{n+1,-}$ are provided by

$$
\begin{aligned}
& \overline{\mathbb{U}}_{i+1 / 2}^{n+1,+}=\frac{1}{\Delta x}\left[\left(b_{l, i+1 / 2}\right)^{+} \Delta t-0\right] \mathbb{U}_{i}^{n}+\frac{1}{\Delta x}\left[\left(u_{1, i+1 / 2}^{\star}\right)^{+} \Delta t-\left(b_{l, i+1 / 2}\right)^{+} \Delta t\right] \mathbb{U}_{i, l}^{\star} \\
& +\frac{1}{\Delta x}\left[\left(b_{r, i+1 / 2}\right)^{+} \Delta t-\left(u_{1, i+1 / 2}^{\star}\right)^{+} \Delta t\right] \mathbb{U}_{i, r}^{\star}+\frac{1}{\Delta x}\left[\frac{\Delta x}{2}-\left(b_{r, i+1 / 2}\right)^{+} \Delta t\right] \mathbb{U}_{i+1}^{n}, \\
& \overline{\mathbb{U}}_{i+1,2}^{n+1,-}=\frac{1}{\Delta x}\left[\left(b_{l, i+1 / 2}\right)^{-} \Delta t+\frac{\Delta x}{2}\right] \mathbb{U}_{i}^{n}+\frac{1}{\Delta x}\left[\left(u_{1, i+1 / 2}^{\star}\right)^{-} \Delta t-\left(b_{l, i+1 / 2}\right)^{-} \Delta t\right] \mathbb{U}_{i, l}^{\star} \\
& +\frac{1}{\Delta x}\left[\left(b_{r, i+1 / 2}\right)^{-} \Delta t-\left(u_{1, i+1 / 2}^{\star}\right)^{-} \Delta t\right] \mathbb{U}_{i, r}^{\star}+\frac{1}{\Delta x}\left[0-\left(b_{r, i+1 / 2}\right)^{-} \Delta t\right] \mathbb{U}_{i+1}^{n},
\end{aligned}
$$

where the involved intermediate quantities come from the intermediate states defined inside $\mathbb{U}_{\mathcal{R}}\left(\frac{x}{t} ; \mathbb{U}_{i}^{e q, n}, \mathbb{U}_{i+1}^{e q, n}\right)$. As usual, we have introduced the notations $\alpha^{+}=\max (\alpha, 0)$ and $\alpha^{-}=\min (\alpha, 0)$.

Second step: Relaxation step. The system

$$
\left\{\begin{array}{l}
\partial_{t} \mathbb{U}=\delta \mathbb{T}(\mathbb{U}), \\
\mathbb{U}_{0}(x)=\overline{\mathbb{U}}_{i}^{n+1} \quad \text { if } \quad x \in\left(x_{i-1 / 2}, x_{i+1 / 2}\right),
\end{array}\right.
$$

is solved in the limit of $\delta$ tending to infinity. On each cell $\left(x_{i-1 / 2}, x_{i+1 / 2}\right)$ the solution of (5.11) is given by

$$
\mathbb{U}_{i}^{e q, n+1}=\mathbb{U}^{e q}\left(\mathcal{N} \overline{\mathbb{U}}_{i}^{n+1} ; \varphi_{i}^{e q, n+1}, a_{i}^{n+1}\right) .
$$

Let us remark that we recover

$$
\Pi_{11, i}^{n+1}=P_{11, i}^{n+1}=\rho_{i}^{n+1} \varepsilon_{11, i}^{n+1}, \quad \Pi_{12, i}^{n+1}=P_{12, i}^{n+1}=\rho_{i}^{n+1} \varepsilon_{12, i}^{n+1}, \quad \Pi_{22, i}^{n+1}=P_{22, i}^{n+1}=\rho_{i}^{n+1} \varepsilon_{22, i}^{n+1} .
$$

Similarly, the quantity $\varphi_{i}^{e q, n+1}$ is also updated as an approximation, at equilibrium, of the integral

$$
\frac{1}{2} \int_{0}^{x_{i}} \rho\left(\xi, t^{n+1}\right) \partial_{\xi} W\left(\xi, t^{n+1}\right) d \xi
$$

A precise discretization of this integral will be clarified later on. follows

Finally, at time $t^{n+1}$, the updated approximate solution $\mathcal{U}_{i}^{n+1}$ is then obtained as

$$
\mathcal{U}_{i}^{n+1}=\mathcal{N} \mathbb{U}_{i}^{e q, n+1}=\mathcal{N} \overline{\mathbb{U}}_{i}^{n+1}
$$

Considering the relations (5.9)-(5.10), the updated state rewrites

$$
\mathcal{U}_{i}^{n+1}=\mathcal{U}_{i-1 / 2}^{n+1,+}+\mathcal{U}_{i+1 / 2}^{n+1,-}
$$

where

$$
\begin{aligned}
& \mathcal{U}_{i+1 / 2}^{n+1,+}=\frac{1}{\Delta x}\left[\left(b_{l, i+1 / 2}\right)^{+} \Delta t-0\right] \mathcal{U}_{i}^{n}+\frac{1}{\Delta x}\left[\left(u_{1, i+1 / 2}^{\star}\right)^{+} \Delta t-\left(b_{l, i+1 / 2}\right)^{+} \Delta t\right] \mathcal{N} \mathbb{U}_{i, l}^{\star} \\
& +\frac{1}{\Delta x}\left[\left(b_{r, i+1 / 2}\right)^{+} \Delta t-\left(u_{1, i+1 / 2}^{\star}\right)^{+} \Delta t\right] \mathcal{N} \mathbb{U}_{i, r}^{\star}+\frac{1}{\Delta x}\left[\frac{\Delta x}{2}-\left(b_{r, i+1 / 2}\right)^{+} \Delta t\right] \mathcal{U}_{i+1}^{n}, \\
& \mathcal{U}_{i+1 / 2}^{n+1,-}=\frac{1}{\Delta x}\left[\left(b_{l, i+1 / 2}\right)^{-} \Delta t+\frac{\Delta x}{2}\right] \mathcal{U}_{i}^{n}+\frac{1}{\Delta x}\left[\left(u_{1, i+1 / 2}^{\star}\right)^{-} \Delta t-\left(b_{l, i+1 / 2}\right)^{-} \Delta t\right] \mathcal{N} \mathbb{U}_{i, l}^{\star} \\
& +\frac{1}{\Delta x}\left[\left(b_{r, i+1 / 2}\right)^{-} \Delta t-\left(u_{1, i+1 / 2}^{\star}\right)^{-} \Delta t\right] \mathcal{N} \mathbb{U}_{i, r}^{\star}+\frac{1}{\Delta x}\left[0-\left(b_{r, i+1 / 2}\right)^{-} \Delta t\right] \mathcal{U}_{i+1}^{n} .
\end{aligned}
$$


The determination of the scheme is achieved as soon as we exhibit a precise definition for $\varphi_{i}^{e q, n}$. Now, since the intermediate states defined by (4.19) only involve the variation of $\varphi$, we have to consider the following approximation:

$$
\varphi_{i+1}^{e q, n}-\varphi_{i}^{e q, n} \simeq \frac{1}{2} \int_{x_{i}}^{x_{i+1}} \rho\left(\xi, t^{n}\right) \partial_{\xi} W\left(\xi, t^{n}\right) d \xi .
$$

As a consequence, we suggest the following formula:

$$
\varphi_{i+1}^{n}-\varphi_{i}^{n}=\frac{1}{4}\left(\rho_{i}^{n}+\rho_{i+1}^{n}\right)\left(W_{i+1}^{n}-W_{i}^{n}\right) .
$$

To conclude this relaxation scheme presentation, let us notice that the scheme (5.9)(5.10) is nothing but a Godunov-type scheme according to the work by Harten, Lax, van Leer [21]. In fact, we easily see that the associated approximate Riemann solver is given by:

$$
\tilde{\mathcal{U}}\left(\frac{x}{t} ; \mathcal{U}_{l}, \mathcal{U}_{r}\right)=\mathcal{N} \mathbb{U}_{\mathcal{R}}\left(\frac{x}{t} ; \mathbb{U}^{e q}\left(\mathcal{U}_{l} ; \varphi_{l}, a_{l}\right), \mathbb{U}^{e q}\left(\mathcal{U}_{r} ; \varphi_{r}, a_{r}\right)\right)
$$

This approximate Riemann solver is made of two intermediate states that we will denote, to simplify the notations

$$
\tilde{\mathcal{U}}_{g}^{\star}=\mathcal{N} \mathbb{U}_{g}^{\star}\left(\frac{x}{\Delta t} ; \mathbb{U}^{e q}\left(\mathcal{U}_{l} ; \varphi_{l}, a_{l}\right), \mathbb{U}^{e q}\left(\mathcal{U}_{r} ; \varphi_{r}, a_{r}\right)\right) \quad \text { with } \quad g=l, r,
$$

where the intermediate states $\mathbb{U}_{l}^{\star}$ and $\mathbb{U}_{r}^{\star}$ are defined in Lemma 4.2. As a consequence, we get

$$
\begin{aligned}
& \mathcal{U}_{i+1 / 2}^{n+1,+}=\int_{0}^{\Delta x / 2} \mathcal{N} \mathbb{U}_{\mathcal{R}}\left(\frac{x}{\Delta t} ; \mathbb{U}^{e q}\left(\mathcal{U}_{i}^{n} ; \varphi_{i}^{e q, n}, a_{i}^{n}\right), \mathbb{U}^{e q}\left(\mathcal{U}_{i+1}^{n} ; \varphi_{i+1}^{n}, a_{i+1}^{n}\right)\right) d x \\
& \mathcal{U}_{i+1 / 2}^{n+1,-}=\int_{-\Delta x / 2}^{0} \mathcal{N} \mathbb{U}_{\mathcal{R}}\left(\frac{x}{\Delta t} ; \mathbb{U}^{e q}\left(\mathcal{U}_{i}^{n} ; \varphi_{i}^{e q, n}, a_{i}^{n}\right), \mathbb{U}^{e q}\left(\mathcal{U}_{i+1}^{n} ; \varphi_{i+1}^{n}, a_{i+1}^{n}\right)\right) d x .
\end{aligned}
$$

\section{Discrete Entropy Inequalities}

The present section is devoted to establishing the nonlinear stability of the scheme (5.15)-(5.16). Our purpose is to prove the discrete entropy inequalities (3.3). The first result concerns a sufficient condition to be stated on the intermediate states to enforce the required stability.

THEOREM 6.1. Assume the intermediate states $\tilde{\mathcal{U}}_{l}^{\star}$ and $\tilde{\mathcal{U}}_{r}^{\star}$ defined by (5.19) belong to $\Omega$ for all $\mathcal{U}_{l, r} \in \Omega$ and satisfy the following entropy estimates

$$
\left\{\begin{array}{l}
s(\varepsilon, v)\left(\tilde{\mathcal{U}}_{l}^{\star}\right) \geq s_{l}, \\
s(\varepsilon, v)\left(\tilde{\mathcal{U}}_{r}^{\star}\right) \geq s_{r}
\end{array}\right.
$$

where

$$
\left\{\begin{array}{l}
s_{l}=s(\varepsilon, v)\left(\mathcal{U}_{l}\right)=s\left(\varepsilon_{l}, v_{l}\right) \\
s_{r}=s(\varepsilon, v)\left(\mathcal{U}_{r}\right)=s\left(\varepsilon_{r}, v_{r}\right)
\end{array}\right.
$$

and $s(\varepsilon, v)=s(\varepsilon, v)(\mathcal{U})$ is given by $(2.14)$. 
In addition, assume the following relations are satisfied

$$
\left\{\begin{array}{l}
\rho_{l}^{\star} u_{1}^{\star}-\rho_{l} u_{1, l}=b_{l}\left(\rho_{l}^{\star}-\rho_{l}\right), \\
\rho_{r}^{\star} u_{1}^{\star}-\rho_{r} u_{1, r}=b_{r}\left(\rho_{r}^{\star}-\rho_{r}\right) .
\end{array}\right.
$$

Assume the CFL condition (5.5) holds. Let $\mathcal{U}_{i}^{n} \in \Omega$ for all $i \in \mathbb{Z}$. Then $\mathcal{U}_{i}^{n+1}$ defined by (5.15)-(5.16) preserves the discrete entropy inequalities (3.3) and the minimum entropy principle (3.4).

Proof.

Arguing [21], the considered scheme will be proved to be entropy preserving as soon as the associated approximate Riemann problem (4.15) - (4.20) leading to (5.15)-(5.16) is established to be entropy compatible. Let us just recall that the approximate Riemann solver $\tilde{\mathcal{U}}_{\mathcal{R}}\left(\frac{x}{\Delta t} ; \mathcal{U}_{l}, \mathcal{U}_{r}\right)$ is said to be entropy compatible as soon as the following inequality holds

$$
\begin{aligned}
\frac{1}{\Delta x} \int_{-\Delta x / 2}^{\Delta x / 2}(\rho F(s(\varepsilon, v)))\left(\tilde{\mathcal{U}}_{\mathcal{R}}\left(\frac{x}{\Delta t} ; \mathcal{U}_{l}, \mathcal{U}_{r}\right)\right) d x \leq & \frac{1}{2}\left(\rho_{l} F\left(s_{l}\right)+\rho_{r} F\left(s_{r}\right)\right) \\
& -\frac{\Delta t}{\Delta x}\left(\rho_{r} F\left(s_{r}\right) u_{1, r}-\rho_{l} F\left(s_{l}\right) u_{1, l}\right) .
\end{aligned}
$$

Now considering the approximate Riemann solver (5.15)-(5.18), we show that the above inequality holds.

Integrating $(\rho F(s(\varepsilon, v)))\left(\tilde{\mathcal{U}}_{\mathcal{R}}\left(\frac{x}{\Delta t} ; \mathcal{U}_{l}, \mathcal{U}_{r}\right)\right)$ over $(-\Delta x / 2, \Delta x / 2)$, we get

$$
\begin{aligned}
& \int_{-\Delta x / 2}^{\Delta x / 2}(\rho F(s(\varepsilon, v)))\left(\tilde{\mathcal{U}}_{\mathcal{R}}\left(\frac{x}{\Delta t} ; \mathcal{U}_{l}, \mathcal{U}_{r}\right)\right) d x=\int_{-\Delta x / 2}^{b_{l} \Delta t} \rho_{l} F\left(s_{l}\right) d x \\
& +\int_{b_{l} \Delta t}^{u_{1}^{\star} \Delta t}(\rho F(s(\varepsilon, v)))\left(\tilde{\mathcal{U}}_{l}^{\star}\right) d x+\int_{u_{1}^{\star} \Delta t}^{b_{r} \Delta t}(\rho F(s(\varepsilon, v)))\left(\tilde{\mathcal{U}}_{r}^{\star}\right) d x+\int_{b_{r} \Delta t}^{\Delta x / 2} \rho_{r} F\left(s_{r}\right) d x .
\end{aligned}
$$

For all strictly decreasing functions $F$, from assumption (6.1), we deduce the following inequalities:

$$
\begin{aligned}
& F\left(s(\varepsilon, v)\left(\tilde{\mathcal{U}}_{l}^{\star}\right)\right) \leq F\left(s_{l}\right), \\
& F\left(s(\varepsilon, v)\left(\tilde{\mathcal{U}}_{r}^{\star}\right)\right) \leq F\left(s_{r}\right) .
\end{aligned}
$$

Therefore equality (6.4) rewrites

$$
\begin{aligned}
\int_{-\Delta x / 2}^{\Delta x / 2}(\rho F(s(\varepsilon, v)))\left(\tilde{\mathcal{U}}_{\mathcal{R}}\left(\frac{x}{\Delta t} ; \mathcal{U}_{l}, \mathcal{U}_{r}\right)\right) d x \leq & \int_{-\Delta x / 2}^{b_{l} \Delta t} \rho_{l} F\left(s_{l}\right) d x+\int_{b_{l} \Delta t}^{u_{1}^{\star} \Delta t} \rho_{l}^{\star} F\left(s_{l}\right) d x \\
& +\int_{u_{1}^{\star} \Delta t}^{b_{r} \Delta t} \rho_{r}^{\star} F\left(s_{r}\right) d x+\int_{b_{r} \Delta t}^{\Delta x / 2} \rho_{r} F\left(s_{r}\right) d x
\end{aligned}
$$

to obtain

$$
\begin{aligned}
\frac{1}{\Delta x} \int_{-\Delta x / 2}^{\Delta x / 2}(\rho F(s(\varepsilon, v)))\left(\tilde{\mathcal{U}}_{\mathcal{R}}\left(\frac{x}{\Delta t} ; \mathcal{U}_{l}, \mathcal{U}_{r}\right)\right) d x \leq & \frac{1}{2}\left(\rho_{l} F\left(s_{l}\right)+\rho_{r} F\left(s_{r}\right)\right) \\
& +\frac{\Delta t}{\Delta x}\left(\rho_{l} b_{l}-\rho_{l}^{\star} b_{l}+\rho_{l}^{\star} u_{1}^{\star}\right) F\left(s_{l}\right) \\
& +\frac{\Delta t}{\Delta x}\left(\rho_{r}^{\star} b_{r}-\rho_{r}^{\star} u_{1}^{\star}-\rho_{r} b_{r}\right) F\left(s_{r}\right) .
\end{aligned}
$$


The required inequality (6.3) easily comes from assumption (6.2). In fact, (6.2) holds for the relaxation method proposed in this paper, it is obtained by performing simple computations from (4.19).

To conclude the proof, we have to establish the minimum entropy principle. Since the function $\mathcal{U} \mapsto \rho F(s(\varepsilon, v))$ is convex, the Jensen's inequality gives

$$
\rho_{i}^{n+1} F\left(s_{i}^{n+1}\right) \leq \frac{1}{\Delta x} \int_{x_{i-1 / 2}}^{x_{i+1 / 2}}(\rho F(s(\varepsilon, v)))\left(\mathcal{U}^{\Delta}\left(x, t^{n}+\Delta t\right)\right) d x,
$$

to get

$$
\begin{aligned}
\rho_{i}^{n+1} F\left(s_{i}^{n+1}\right) \leq & \frac{1}{\Delta x} \int_{x_{i-1 / 2}}^{x_{i}}(\rho F(s(\varepsilon, v)))\left(\tilde{\mathcal{U}}_{\mathcal{R}}\left(\frac{x-x_{i-1 / 2}}{\Delta t}, \mathcal{U}_{i-1}^{n}, \mathcal{U}_{i}^{n}\right)\right) d x \\
& +\frac{1}{\Delta x} \int_{x_{i}}^{x_{i+1 / 2}}(\rho F(s(\varepsilon, v)))\left(\tilde{\mathcal{U}}_{\mathcal{R}}\left(\frac{x-x_{i+1 / 2}}{\Delta t}, \mathcal{U}_{i}^{n}, \mathcal{U}_{i+1}^{n}\right)\right) d x
\end{aligned}
$$

By averaging (6.1), we have

$$
s(\varepsilon, v)\left(\tilde{\mathcal{U}}_{\mathcal{R}}\left(\frac{x-x_{i+1 / 2}}{\Delta t}, \mathcal{U}_{i}^{n}, \mathcal{U}_{i+1}^{n}\right)\right) \geq \min \left(s_{i}^{n}, s_{i+1}^{n}\right) .
$$

Since $F$ is a strictly decreasing function, from (6.5) we immediately deduce

$$
\rho_{i}^{n+1} F\left(s_{i}^{n+1}\right) \leq F\left(\min \left(s_{i-1}^{n}, s_{i}^{n}, s_{i+1}^{n}\right)\right) \frac{1}{\Delta x} \int_{x_{i-1 / 2}}^{x_{i+1 / 2}} \rho^{\Delta}\left(x, t^{n}+\Delta t\right) d x .
$$

Because $\rho_{i}^{n+1}=\frac{1}{\Delta x} \int_{x_{i-1 / 2}}^{x_{i+1 / 2}} \rho^{\Delta}\left(x, t^{n}+\Delta t\right) d x$, we get

$$
F\left(s_{i}^{n+1}\right) \leq F\left(\min \left(s_{i-1}^{n}, s_{i}^{n}, s_{i+1}^{n}\right)\right)
$$

or equivalently

$$
s_{i}^{n+1} \geq \min \left(s_{i-1}^{n}, s_{i}^{n}, s_{i+1}^{n}\right) .
$$

The proof is thus complete.

Our goal is now to show that the proposed intermediates states $\mathcal{U}_{l}^{\star}$ and $\mathcal{U}_{r}^{\star}$ satisfy the stability condition (6.1). To address such an issue, we need some following technical lemmas.

The first lemma exhibits suitable invariants across the wave speeds $b_{l}$ and $b_{r}$ that are useful for establishing the stability condition (6.1).

LEMma 6.2. The quantities $\Pi_{11, g}+\left(a_{g}\right)^{2} / \rho_{g}, \quad \varepsilon_{11, g}-\left(\Pi_{11, g}\right)^{2} /\left(a_{g}\right)^{2}, \quad \varepsilon_{12, g}-$ $\Pi_{11, g} \Pi_{12, g} /\left(a_{g}\right)^{2}$ and $\varepsilon_{22, g}-\left(\Pi_{12, g}\right)^{2} /\left(a_{g}\right)^{2}$ are invariant across the wave speeds $b_{l}=$ $u_{1, l}-a_{l} / \rho_{l}$ and $b_{r}=u_{1, r}+a_{r} / \rho_{r}$,

$$
\begin{aligned}
& \Pi_{11, g}^{\star}+\frac{\left(a_{g}\right)^{2}}{\rho_{g}^{\star}}=\Pi_{11, g}+\frac{\left(a_{g}\right)^{2}}{\rho_{g}}, \\
& \varepsilon_{11, g}^{\star}-\frac{\left(\Pi_{11, g}^{\star}\right)^{2}}{\left(a_{g}\right)^{2}}=\varepsilon_{11, g}-\frac{\left(\Pi_{11, g}\right)^{2}}{\left(a_{g}\right)^{2}}, \\
& \varepsilon_{12, g}^{\star}-\frac{\Pi_{11, g}^{\star} \Pi_{12}^{\star}}{\left(a_{g}\right)^{2}}=\varepsilon_{12, g}-\frac{\Pi_{11, g} \Pi_{12, g}}{\left(a_{g}\right)^{2}}, \\
& \varepsilon_{22, g}^{\star}-\frac{\left(\Pi_{12}^{\star}\right)^{2}}{\left(a_{g}\right)^{2}}=\varepsilon_{22, g}-\frac{\left(\Pi_{12, g}\right)^{2}}{\left(a_{g}\right)^{2}}
\end{aligned}
$$


where $g$ denotes either $l$ or $r, \Pi_{11, g}^{\star}, \Pi_{12}^{\star}$ and $\Pi_{22, g}^{\star}$ being given by (4.19).

Proof. The proposed quantities are non-trivial Riemann invariants shared by the eigenvalues $u_{1} \pm a / \rho$ already exhibited in (4.22). They are known as strong invariants.

We now introduce the following mapping

$$
f:\left(\varepsilon_{11}, \varepsilon_{12}, \varepsilon_{22}, v\right) \mapsto\left(I, X_{11}, X_{12}, X_{22}\right),
$$

with

$$
\left\{\begin{array}{l}
I=P_{11}+a^{2} v \\
X_{11}=\varepsilon_{11}-\frac{\left(P_{11}\right)^{2}}{a^{2}}, \\
X_{12}=\varepsilon_{12}-\frac{P_{11} P_{12}}{a^{2}}, \\
X_{22}=\varepsilon_{22}-\frac{\left(P_{12}\right)^{2}}{a^{2}}
\end{array}\right.
$$

where $a$ is a real number and $\boldsymbol{P}$ is assumed to satisfy $\boldsymbol{P}=\frac{1}{v} \varepsilon$.

The mapping $f$ enables us to do a suitable change of variables for the entropy $s(\varepsilon, v)$. In this goal, we have the following lemma.

LEMma 6.3. The change of variables $f:\left(\varepsilon_{11}, \varepsilon_{12}, \varepsilon_{22}, v\right) \mapsto\left(I, X_{11}, X_{12}, X_{22}\right)$ is one-toone under the Whitham subcharacteristic condition

$$
\frac{\left(v^{2} a^{2}-\varepsilon_{11}\right)\left(v^{2} a^{2}-3 \varepsilon_{11}\right)}{v^{4} a^{2}}>0 .
$$

Proof. The Jacobian of the change of variables (6.10)-(6.11) reads

$$
J_{f}=\frac{\left(v^{2} a^{2}-\varepsilon_{11}\right)\left(v^{2} a^{2}-3 \varepsilon_{11}\right)}{v^{4} a^{2}} .
$$

The non-vanishing condition for $J_{f}$ that makes $f$ invertible is achieved whenever the relation (6.12) is satisfied.

With the bijection $f$, the entropy can be rewritten as

$$
\begin{aligned}
s(\varepsilon, v) & =s\left(f^{-1}\left(I, X_{11}, X_{12}, X_{22}\right)\right) \\
& =S\left(I(\varepsilon, v), X_{11}(\varepsilon, v), X_{12}(\varepsilon, v), X_{22}(\varepsilon, v)\right),
\end{aligned}
$$

where $S=s f^{-1}$.

We now introduce the following space

$$
\omega=\left\{\Pi \in \mathcal{M}_{3}(\mathbb{R}) \text { such that } \Pi_{33}>0, \Pi_{13}=\Pi_{23}=0\right\},
$$

where $\mathcal{M}_{3}(\mathbb{R})$ is the space of $3 \times 3$ matrices whose entries are real numbers.

The next lemma is connected to Lemma 6.3 by the following remark. Since the intermediate states generated by the relaxation method exposed in this paper are artificial, we are not sure that their pressure can be rewritten in term of the equation of state, i.e. we are not sure that the relations $\Pi_{g}^{\star}=\frac{1}{v_{g}^{\star}} \varepsilon_{g}^{\star}$ hold, where $g=l, r$. Therefore 
we can let the pressure matrix $\boldsymbol{P}$ of a state to be independent from the internal energy matrix $\varepsilon$ and the specific volume $v$. In this way, we can define the variable $\overline{\boldsymbol{\Pi}}$ close to the variable $\boldsymbol{P}$. Then the functions $I, X_{11}, X_{12}$ and $X_{22}$ can be extended into $\bar{I}, \bar{X}_{11}$, $\bar{X}_{12}$ and $\bar{X}_{22}$ on all the space $\omega$ by

$$
\left\{\begin{array}{c}
\bar{I}(\varepsilon, v, \overline{\mathbf{\Pi}})=\Pi_{11}+a^{2} v, \quad \bar{X}_{11}(\varepsilon, v, \bar{\Pi})=\varepsilon_{11}-\frac{\Pi_{11}^{2}}{a^{2}}, \\
\bar{X}_{12}(\varepsilon, v, \bar{\Pi})=\varepsilon_{12}-\frac{\Pi_{11} \Pi_{12}}{a^{2}}, \bar{X}_{22}(\varepsilon, v, \bar{\Pi})=\varepsilon_{22}-\frac{\Pi_{12}^{2}}{a^{2}},
\end{array}\right.
$$

which coincide with $I, X_{11}, X_{12}$ and $X_{22}$ when $\bar{\Pi}=\boldsymbol{P}=\boldsymbol{P}(\varepsilon, v)$ for given $\boldsymbol{\varepsilon}, v$ :

$$
\left\{\begin{array}{c}
\bar{I}(\varepsilon, v, \boldsymbol{P})=P_{11}+a^{2} v=I(\varepsilon, v), \quad \bar{X}_{11}(\varepsilon, v, \boldsymbol{P})=\varepsilon_{11}-\frac{P_{11}^{2}}{a^{2}}=X_{11}(\varepsilon, v), \\
\bar{X}_{12}(\varepsilon, v, \boldsymbol{P})=\varepsilon_{12}-\frac{P_{11} P_{12}}{a^{2}}=X_{12}(\varepsilon, v) \bar{X}_{22}(\varepsilon, v, \boldsymbol{P})=\varepsilon_{22}-\frac{P_{12}^{2}}{a^{2}}=X_{22}(\varepsilon, v) .
\end{array}\right.
$$

Note that only the components $\bar{\Pi}_{11}$ and $\bar{\Pi}_{12}$ of the matrix $\bar{\Pi}$ appear in the definition of the functions $\bar{I}, \bar{X}_{11}, \bar{X}_{12}$ and $\bar{X}_{22}$. Therefore, the derivatives with respect to $\bar{\Pi}$ are actually derivatives with respect to the vector $\left(\begin{array}{l}\bar{\Pi}_{11} \\ \bar{\Pi}_{12}\end{array}\right)$ abusively denoted again $\bar{\Pi}$.

Finally, a function

$$
\bar{S}\left(\bar{I}(\varepsilon, v, \overline{\mathbf{\Pi}}), \bar{X}_{11}(\varepsilon, v, \overline{\mathbf{\Pi}}), \bar{X}_{12}(\varepsilon, v, \overline{\mathbf{\Pi}}), \bar{X}_{22}(\varepsilon, v, \overline{\mathbf{\Pi}})\right)
$$

can be defined, related to the function $S$ by

$$
\begin{aligned}
s(\varepsilon, v) & =S\left(I(\varepsilon, v), X_{11}(\varepsilon, v), X_{12}(\varepsilon, v), X_{22}(\varepsilon, v)\right) \\
& =\left.\bar{S}\left(\bar{I}(\varepsilon, v, \overline{\mathbf{\Pi}}), \bar{X}_{11}(\varepsilon, v, \mathbf{\Pi}), \bar{X}_{12}(\varepsilon, v, \overline{\mathbf{\Pi}}), \bar{X}_{22}(\varepsilon, v, \overline{\mathbf{\Pi}})\right)\right|_{\overline{\mathbf{\Pi}}=\boldsymbol{P}(\varepsilon, v)}
\end{aligned}
$$

Although the new pressure-like matrix variable $\overline{\mathbf{\Pi}}$ belongs to the whole space $\omega$, one can exhibit the following result which is a key idea for establishing the stability condition (6.1) of the two intermediate states solvers proposed in this paper.

LEMma 6.4. The maximization principle on the specific entropy holds

$$
\begin{aligned}
s(\varepsilon, v) & =S\left(I(\varepsilon, v), X_{11}(\varepsilon, v), X_{12}(\varepsilon, v), X_{22}(\varepsilon, v)\right) \\
& =\left.\bar{S}\left(\bar{I}(\varepsilon, v, \overline{\mathbf{\Pi}}), \bar{X}_{11}(\varepsilon, v, \overline{\mathbf{\Pi}}), \bar{X}_{12}(\varepsilon, v, \overline{\mathbf{\Pi}}), \bar{X}_{22}(\varepsilon, v, \overline{\mathbf{\Pi}})\right)\right|_{\overline{\mathbf{\Pi}}=\boldsymbol{P}(\varepsilon, v)} \\
& =\max _{\overline{\mathbf{\Pi}} \in \omega} \bar{S}\left(\bar{I}(\varepsilon, v, \overline{\mathbf{\Pi}}), \bar{X}_{11}(\varepsilon, v, \overline{\mathbf{\Pi}}), \bar{X}_{12}(\varepsilon, v, \overline{\mathbf{\Pi}}), \bar{X}_{22}(\varepsilon, v, \overline{\mathbf{\Pi}})\right)
\end{aligned}
$$

where the pressure matrix $\boldsymbol{P}$ is related to the given internal energy matrix $\boldsymbol{\varepsilon}$ and the specific volume $v$ by $\boldsymbol{P}=\frac{1}{v} \varepsilon$.

Proof. Let $Q$ and $\bar{W}$ be the vectors $Q=\left(\begin{array}{c}\varepsilon_{11} \\ \varepsilon_{12} \\ \varepsilon_{22} \\ v\end{array}\right), \bar{W}=\left(\begin{array}{c}\bar{I}_{11} \\ \bar{X}_{11} \\ \bar{X}_{12} \\ \bar{X}_{22}\end{array}\right)$. For given $Q$ and pressure $\left(\begin{array}{l}\bar{\Pi}_{11} \\ \bar{\Pi}_{12}\end{array}\right)$, the vectors $Q$ and $\bar{W}$ are related by the equations (6.14). Thanks to 
the bijection $(6.10)-(6.11)$, there exists a vector $\tilde{Q}=\left(\begin{array}{c}\tilde{\varepsilon}_{11} \\ \tilde{\varepsilon}_{12} \\ \tilde{\varepsilon}_{22} \\ \tilde{v}\end{array}\right)$ such that $\bar{W}=f(\tilde{Q})$ :

$$
\left\{\begin{array}{c}
\bar{I}=P_{11}(\tilde{\varepsilon}, \tilde{v})+a^{2} \tilde{v}=I(\tilde{\varepsilon}, \tilde{v}), \quad \bar{X}_{11}=\tilde{\varepsilon}_{11}-\frac{P_{11}^{2}(\tilde{\varepsilon}, \tilde{v})}{a^{2}}=X_{11}(\tilde{\varepsilon}, \tilde{v}), \\
\bar{X}_{12}=\tilde{\varepsilon}_{12}-\frac{P_{11}(\tilde{\varepsilon}, \tilde{v}) P_{12}(\tilde{\varepsilon}, \tilde{v})}{a^{2}}=X_{12}(\tilde{\varepsilon}, \tilde{v}), \bar{X}_{22}=\tilde{\varepsilon}_{22}-\frac{P_{12}^{2}(\tilde{\varepsilon}, \tilde{v})}{a^{2}}=X_{22}(\tilde{\varepsilon}, \tilde{v}) .
\end{array}\right.
$$

Now for given $\boldsymbol{\varepsilon}, v$ and for all $\overline{\boldsymbol{\Pi}} \in \omega$, the quantity

$$
\bar{S}\left(\bar{I}(\varepsilon, v, \overline{\mathbf{\Pi}}), \bar{X}_{11}(\varepsilon, v, \overline{\mathbf{\Pi}}), \bar{X}_{12}(\varepsilon, v, \overline{\mathbf{\Pi}}), \bar{X}_{22}(\varepsilon, v, \overline{\mathbf{\Pi}})\right)
$$

rewrites

$$
\begin{aligned}
\bar{S}\left(\bar{I}(\varepsilon, v, \overline{\mathbf{\Pi}}), \bar{X}_{11}(\varepsilon, v, \overline{\mathbf{\Pi}}), \bar{X}_{12}(\varepsilon, v, \overline{\mathbf{\Pi}}), \bar{X}_{22}(\varepsilon, v, \overline{\mathbf{\Pi}})\right) & =\bar{S}(\bar{W}(\overline{\mathbf{\Pi}})) \\
& =s(\tilde{Q}) \\
& =s(\tilde{Q}(\bar{W}(\overline{\mathbf{\Pi}}))) .
\end{aligned}
$$

Then the derivative of $\bar{S}$ with respect to $\bar{\Pi}$ is given by the chain rule

$$
\begin{aligned}
\frac{\partial \bar{S}}{\partial \overline{\mathbf{\Pi}}} & \left(\bar{I}(\varepsilon, v, \overline{\mathbf{\Pi}}), \bar{X}_{11}(\varepsilon, v, \overline{\mathbf{\Pi}}), \bar{X}_{12}(\varepsilon, v, \overline{\mathbf{\Pi}}), \bar{X}_{22}(\varepsilon, v, \overline{\mathbf{\Pi}})\right) \\
& =\frac{\partial s}{\partial Q}(\tilde{Q}) \cdot \frac{\partial \tilde{Q}}{\partial W}(\bar{W}) \cdot \frac{\partial \bar{W}}{\partial \overline{\mathbf{\Pi}}}(\overline{\mathbf{\Pi}}) \\
& =\frac{\partial s}{\partial Q}(\tilde{Q}) \cdot\left(\frac{\partial W}{\partial \tilde{Q}}\right)^{-1}(\bar{W}) \cdot \frac{\partial \bar{W}}{\partial \overline{\mathbf{\Pi}}}(\overline{\mathbf{\Pi}})
\end{aligned}
$$

which can be rewritten as

$$
\begin{aligned}
& \frac{\partial \bar{S}}{\partial \bar{\Pi}}\left(\bar{I}(\varepsilon, v, \bar{\Pi}), \bar{X}_{11}(\varepsilon, v, \bar{\Pi}), \bar{X}_{12}(\varepsilon, v, \bar{\Pi}), \bar{X}_{22}(\varepsilon, v, \bar{\Pi})\right)= \\
& =\left(\frac{2\left(\tilde{v} \tilde{\varepsilon}_{12} \bar{\Pi}_{12}-\tilde{v} \tilde{\varepsilon}_{22} \bar{\Pi}_{11}+\tilde{\varepsilon}_{11} \tilde{\varepsilon}_{22}-\tilde{\varepsilon}_{12}^{2}\right)}{a^{2} \tilde{v}\left(\tilde{\varepsilon}_{11} \tilde{\varepsilon}_{22}-\tilde{\varepsilon}_{12}^{2}\right)} ;-\frac{2\left(-\tilde{\varepsilon}_{12} \bar{\Pi}_{11}+\tilde{\varepsilon}_{11} \bar{\Pi}_{12}\right)}{a^{2}\left(\tilde{\varepsilon}_{11} \tilde{\varepsilon}_{22}-\tilde{\varepsilon}_{12}^{2}\right)}\right) .
\end{aligned}
$$

The gradient $\frac{\partial \bar{S}}{\partial \bar{\Pi}}$ vanish at $\left(\begin{array}{l}\bar{\Pi}_{11}=\frac{\tilde{\varepsilon}_{11}}{\tilde{v}} \\ \bar{\Pi}_{12}=\frac{\tilde{\varepsilon}_{12}}{\tilde{v}}\end{array}\right)$. At these values the Hessian is given by

$$
\frac{\partial^{2} \bar{S}}{\partial \overline{\mathbf{\Pi}}^{2}}=\left(\begin{array}{cc}
-\frac{2 \tilde{\varepsilon}_{22}}{a^{2}\left(\tilde{\varepsilon}_{11} \tilde{\varepsilon}_{22}-\tilde{\varepsilon}_{12}^{2}\right)} & \frac{2 \tilde{\varepsilon}_{12}}{a^{2}\left(\tilde{\varepsilon}_{11} \tilde{\varepsilon}_{22}-\tilde{\varepsilon}_{12}^{2}\right)} \\
\frac{2 \tilde{\varepsilon}_{12}}{a^{2}\left(\tilde{\varepsilon}_{11} \tilde{\varepsilon}_{22}-\tilde{\varepsilon}_{12}^{2}\right)} & -\frac{2 \tilde{\varepsilon}_{11}}{a^{2}\left(\tilde{\varepsilon}_{11} \tilde{\varepsilon}_{22}-\tilde{\varepsilon}_{12}^{2}\right)}
\end{array}\right)
$$

Since the determinant of this Hessian

$$
\frac{4}{a^{2}\left(\tilde{\varepsilon}_{11} \tilde{\varepsilon}_{22}-\tilde{\varepsilon}_{12}^{2}\right)}>0
$$

and its trace

$$
-\frac{2\left(\tilde{\varepsilon}_{22}+\tilde{\varepsilon}_{11}\right)}{a^{2}\left(\tilde{\varepsilon}_{11} \tilde{\varepsilon}_{22}-\tilde{\varepsilon}_{12}^{2}\right)}<0
$$


the Hessian is definite negative. As a consequence $\left(\begin{array}{c}\frac{\tilde{\varepsilon}_{11}}{\tilde{v}} \\ \frac{\tilde{\varepsilon}_{12}}{\tilde{v}}\end{array}\right)$ is a maximum of the mapping $\overline{\mathbf{\Pi}} \mapsto \bar{S}(\bar{W}(\overline{\mathbf{\Pi}}))$

In fact for given $\varepsilon, v$, only $\left(\begin{array}{c}\frac{\varepsilon_{11}}{v} \\ \frac{\varepsilon_{12}}{v}\end{array}\right)$ is the maximum of $\overline{\mathbf{\Pi}} \mapsto \bar{S}(\bar{W}(\overline{\mathbf{\Pi}}))$. Indeed $\varepsilon, v$ satisfy

$$
\begin{gathered}
I(\varepsilon, v)-P_{11}=a^{2} v, \quad X_{11}(\varepsilon, v)+\frac{P_{11}^{2}}{a^{2}}=\varepsilon_{11}, \\
X_{12}(\varepsilon, v)+\frac{P_{11} P_{12}}{a^{2}}=\varepsilon_{12}, X_{22}(\varepsilon, v)+\frac{P_{12}^{2}}{a^{2}}=\varepsilon_{22} .
\end{gathered}
$$

Setting $\overline{\boldsymbol{\Pi}}=\boldsymbol{P}(\tilde{\varepsilon}, \tilde{v})$ in $(6.14)$, we get

$$
\begin{array}{cc}
\bar{I}-P_{11}(\tilde{\boldsymbol{\varepsilon}}(\bar{W}), \tilde{v}(\bar{W}))=a^{2} v, & \bar{X}_{11}+\frac{P_{11}^{2}(\tilde{\boldsymbol{\varepsilon}}(\bar{W}), \tilde{v}(\bar{W}))}{a^{2}}=\varepsilon_{11}, \\
\bar{X}_{12}+\frac{P_{11}(\tilde{\boldsymbol{\varepsilon}}(\bar{W}), \tilde{v}(\bar{W})) P_{12}(\tilde{\boldsymbol{\varepsilon}}(\bar{W}), \tilde{v}(\bar{W}))}{a^{2}}=\varepsilon_{12}, \bar{X}_{22}+\frac{P_{12}^{2}(\tilde{\boldsymbol{\varepsilon}}(\bar{W}), \tilde{v}(\bar{W}))}{a^{2}}=\varepsilon_{22} .
\end{array}
$$

The set of equations (6.16) can be rewritten as the nonlinear system $g(\bar{W})=0$ where $\bar{W} \mapsto g(\bar{W})$ is obvious. The Jacobian of $g$ with respect to $\bar{W}$ is

$$
J_{g}=\frac{\tilde{v}^{4}(\bar{W}) a^{2}}{\left(\tilde{v}(\bar{W})^{2} a^{2}-\tilde{\varepsilon}_{11}(\bar{W})\right)\left(\tilde{v}^{2}(\bar{W}) a^{2}-3 \tilde{\varepsilon}_{11}(\bar{W})\right)},
$$

which does not vanish thanks to the Whitham subcharacteristic condition (6.12). Then for given $\varepsilon, v$, the set of equations (6.16) is invertible and its solution is $I, X_{11}, X_{12}$, $X_{22}$. Hence $W=\bar{W}$, and $\tilde{\varepsilon}=\varepsilon, \tilde{v}=v$. Therefore only $\left(\begin{array}{c}\frac{\varepsilon_{11}}{v} \\ \frac{\varepsilon_{12}}{v}\end{array}\right)$ is the maximum of the mapping $\overline{\mathbf{\Pi}} \mapsto \bar{S}(\bar{W}(\overline{\mathbf{\Pi}}))$. The lemma is thus proved.

Thanks to the maximum principle (6.15), we conclude this section by establishing the stability condition (6.1). Indeed, we have the following easy sequence of relations

$$
\begin{aligned}
s(\varepsilon, v)\left(\tilde{\mathcal{U}}_{l}^{\star}\right) & =\left.\bar{S}\left(\bar{I}(\varepsilon, v, \overline{\mathbf{\Pi}})\left(\tilde{\mathcal{U}}_{l}^{\star}\right), \bar{X}_{11}(\varepsilon, v, \overline{\mathbf{\Pi}})\left(\tilde{\mathcal{U}}_{l}^{\star}\right), \bar{X}_{12}(\varepsilon, v, \overline{\mathbf{\Pi}})\left(\tilde{\mathcal{U}}_{l}^{\star}\right), \bar{X}_{22}(\varepsilon, v, \overline{\mathbf{\Pi}})\left(\tilde{\mathcal{U}}_{l}^{\star}\right)\right)\right|_{\overline{\boldsymbol{\Pi}}=\boldsymbol{P}(\varepsilon, v)\left(\tilde{\mathcal{U}}_{l}^{\star}\right)} \\
& =\max _{\overline{\mathbf{\Pi}} \in \omega} \bar{S}\left(\bar{I}\left(\tilde{\varepsilon}_{l}^{\star}, \tilde{v}_{l}^{\star}, \overline{\mathbf{\Pi}}\right), \bar{X}_{11}\left(\tilde{\varepsilon}_{l}^{\star}, \tilde{v}_{l}^{\star}, \overline{\mathbf{\Pi}}\right), \bar{X}_{12}\left(\tilde{\varepsilon}_{l}^{\star}, \tilde{v}_{l}^{\star}, \overline{\mathbf{\Pi}}\right), \bar{X}_{22}\left(\tilde{\varepsilon}_{l}^{\star}, \tilde{v}_{l}^{\star}, \overline{\mathbf{\Pi}}\right)\right) \\
& \geq \bar{S}\left(\bar{I}\left(\tilde{\varepsilon}_{l}^{\star}, \tilde{v}_{l}^{\star}, \tilde{\bar{\Pi}}_{l}^{\star}\right), \bar{X}_{11}\left(\tilde{\varepsilon}_{l}^{\star}, \tilde{v}_{l}^{\star}, \tilde{\bar{\Pi}}_{l}^{\star}\right), \bar{X}_{12}\left(\tilde{\varepsilon}_{l}^{\star}, \tilde{v}_{l}^{\star}, \tilde{\bar{\Pi}}_{l}^{\star}\right), \bar{X}_{22}\left(\tilde{\varepsilon}_{l}^{\star}, \tilde{v}_{l}^{\star}, \tilde{\bar{\Pi}}_{l}^{\star}\right)\right),
\end{aligned}
$$

Next by involving Lemma 6.2 , we deduce the following equalities

$$
\begin{aligned}
& \bar{S}\left(\bar{I}\left(\tilde{\varepsilon}_{l}^{\star}, \tilde{v}_{l}^{\star}, \tilde{\bar{\Pi}}_{l}^{\star}\right), \bar{X}_{11}\left(\tilde{\varepsilon}_{l}^{\star}, \tilde{v}_{l}^{\star}, \tilde{\bar{\Pi}}_{l}^{\star}\right), \bar{X}_{12}\left(\tilde{\varepsilon}_{l}^{\star}, \tilde{v}_{l}^{\star}, \tilde{\bar{\Pi}}_{l}^{\star}\right), \bar{X}_{22}\left(\tilde{\varepsilon}_{l}^{\star}, \tilde{v}_{l}^{\star}, \tilde{\bar{\Pi}}_{l}^{\star}\right)\right)= \\
& \bar{S}\left(\bar{I}\left(\varepsilon_{l}, v_{l}, \overline{\mathbf{\Pi}}_{l}\right), \bar{X}_{11}\left(\varepsilon_{l}, v_{l}, \overline{\mathbf{\Pi}}_{l}\right), \bar{X}_{12}\left(\varepsilon_{l}, v_{l}, \overline{\mathbf{\Pi}}_{l}\right), \bar{X}_{22}\left(\varepsilon_{l}, v_{l}, \overline{\mathbf{\Pi}}_{l}\right)\right)= \\
& s(\varepsilon, v)\left(\mathcal{U}_{l}\right),
\end{aligned}
$$

and the first estimation (6.1) is established. Next, similar arguments immediately give the second estimation $s(\varepsilon, v)\left(\tilde{\mathcal{U}}_{r}^{\star}\right) \geq s(\varepsilon, v)\left(\mathcal{U}_{r}\right)$. Therefore the relaxation scheme proposed in this paper is entropy preserving. 


\begin{tabular}{|c|c|c|c|c|c|c|c|}
\hline & $\rho$ & $\rho u_{1}$ & $\rho u_{2}$ & $\rho e_{11}$ & $\rho e_{12}$ & $\rho e_{22}$ & $\rho e_{33}$ \\
\hline \hline Uniform state & 1 & 0 & 0 & 25 & 7 & 9 & 8 \\
\hline
\end{tabular}

TABLE 7.1. Uniform state data for a gaussian-type source terms test cases.

\begin{tabular}{|c|c|c|c|c|c|c|c|}
\hline & $\rho$ & $\rho u_{1}$ & $\rho u_{2}$ & $\rho e_{11}$ & $\rho e_{12}$ & $\rho e_{22}$ & $\rho e_{33}$ \\
\hline \hline Left state & 1 & -4 & 0 & 25 & 7 & 9 & 8 \\
\hline Right state & 1 & 4 & 0 & 25 & 7 & 9 & 8 \\
\hline
\end{tabular}

TABLE 7.2. Two rarefaction waves state data for a gaussian-type source term test case.

\section{Numerical Tests}

We now turn to the application of the scheme designed in this paper to certain pertinent test cases. These tests are shock tube types in $1 \mathrm{D}$ setting on the interval $[0,4]$ along the $x$-axis and the discontinuity is located at $x=2$. The numerical solution is computed with 4000 points and the CFL condition is always 0.5 for one dimensional test cases. In the two dimensional setting, the CFL condition is 0.25 and the hierarchy of meshes consisting of 101 by 101 cells, 202 by 202 cells, and 404 by 404 cells, is used. Finally, computational domains are chosen sufficiently large so that boundary conditions do not influence simulations.

\subsection{Uniform plasma state with a gaussian-type source term}

The initial data is given in Table 7.1. The source term is of gaussian-type, given by

$$
W(x, t)=25 \exp \left(-200(x-2)^{2}\right) .
$$

This expression is typical one of the laser beam used in ICF. In Figure 7.1 is shown the density $\rho$ computed at time 0.1 by the relaxation scheme proposed in this work. The density is no longer uniform as in the case where the source term is not used. The solution is symmetric according to the center $x=2$ where the source term is localized. A hole is created which is a manifestation of expulsion of particles due to the force derived from the gradient of the potential $W$. Entropy is produced as displayed in Figure 7.1, especially in the hole.

\subsection{Two rarefaction waves problem with a gaussian-type source term}

The inputs of this test are given in Table 7.2 while the source term used is of gaussian-type, provided again by (7.1). When the source term does no longer exist, this test consists of two rarefaction waves propagating in opposite sides with the same speed, already discussed in $[5,31]$. This severe test leading to vacuum state if the speed is very large, is well-treated by the new scheme proposed in this paper although the presence of the source term, as displayed in Figure 7.2.

\subsection{Nonuniform plasma state with a gaussian-type source term in $2 \mathrm{D}$ geometry}

We consider a square of size $100 \mu \mathrm{m}$ containing a quasineutral plasma. The density of electrons is $n_{e_{0}}=10^{27} \mathrm{~m}^{-3}$ while the charge number of ions is $Z=4$ and the atomic mass is 8 . The initial temperature of the plasma is $T_{e_{0}}=2.3 \times 10^{7} \mathrm{~K}$. This plasma is heated by a laser beam at the center of the square. The laser hot spot is round with its center localized at $\left(x_{0}, y_{0}\right)=(50 \mu \mathrm{m}, 50 \mu \mathrm{m})$, and its radius is $R_{0}=10 \mu \mathrm{m}$. The laser 
light wavelength is $0.35 \mu \mathrm{m}$. The laser light is assumed to be a gaussian function with its maximum intensity $I_{0}=3.89 \times 10^{19} \mathrm{Watt} / \mathrm{m}^{2}$, so that the electron quiver energy writes

$$
W(x, y, t)=\frac{Z}{m_{\text {ion }}} I_{0} \exp \left(-\left(\frac{x-x_{0}}{R_{0}}\right)^{2}-\left(\frac{y-y_{0}}{R_{0}}\right)^{2}\right),
$$

where $m_{\text {ion }}$ is the mass of ions. Finally, the laser light is supposed to be polarized linearly along the $x$-direction leading to the anisotropic quiver energy tensor

$$
\boldsymbol{W}=W(x, y, t) \boldsymbol{e}_{1} \otimes \boldsymbol{e}_{1},
$$

to add to the proper isotropic internal energy tensor of electrons when the laser-matter interaction is turned off, in order to obtain the tensor $\boldsymbol{P}$.

For clarity, we denote by HLLC-splitting scheme the scheme aiming to approximate the solution of (2.1), which is based on splitting strategy where the numerical fluxes are computed by either the HLLC scheme [31] or the relaxation method (4.3) omitting the source term $\varphi^{e q}$, and the source terms are computed by Euler explicit method.

Densities are displayed in Figure 7.3. The mesh refinement shows that the solutions computed by the two schemes agree, particularly the new scheme is well-suited to compute the solution.

\subsection{More realistic simulation in $2 \mathrm{D}$ geometry}

An advanced simulation is proposed in adding the effect of the inverse bremsstrahlung absorption in the above test case. In inverse bremsstrahlung absorption, a photon (or light wave) moves an electron past a nucleus. The interaction with the nucleus randomizes the motion of the electron, which has the effect of extracting energy from the light $[1,17]$. The process of inverse bremsstrahlung in the anisotropic plasma considered in this paper can be modelled by adding a source term $2 \nu_{T} \rho \boldsymbol{W}$ in the energy equation. This yields

$$
\partial_{t}(\rho \boldsymbol{u} \otimes \boldsymbol{u}+\boldsymbol{P})+\nabla \cdot(\rho \boldsymbol{H} \otimes \boldsymbol{u})^{S}=-(\rho \boldsymbol{\nabla} W \otimes \boldsymbol{u})^{S}+2 \nu_{T} \rho \boldsymbol{W} .
$$

For simplicity, the absorption coefficient $\nu_{T}$ is set to 1 . The reader is referred to $[18,29,30]$ for more details on computation of inverse bremsstrahlung coefficient absorption. The same geometry and simulation parameters as in the test case 7.3 are used. Temperature, density and entropy at the initial time, $25 \mathrm{ps}, 50 \mathrm{ps}$ are shown in Figure 7.4. Effects of plasma heating due to laser light are obviously displayed. The more larger the temperature becomes in the center of the plasma, the more particles are expulsed from this center. Figure 7.5 shows the state of the plasma after $50 \mathrm{ps}$ simulation time.

\section{Conclusion}

In the present paper, a relaxation-type scheme has been built to approximate weak solutions of the Ten-Moments equations with source terms. Concerning stability issue of the scheme, to prove discrete entropy inequalities, we derive a new strategy based on local minimum entropy principle. Moreover, the procedure is well-suited to show the entropy skills of the HLLC scheme already derived for Ten-Moments omitting source terms in another previous paper [31]. Numerical experiments have been also addressed to show the robustness of the scheme.

Forthcoming works will concern the establishment of local minimum entropy principle for advanced model of laser-matter interaction in ICF on one side. This model will be a coupling of Ten-Moments equations with radiative transfer and magnetic fields 
generation. On another side the establishment of local minimum entropy principle will be investigated for Saint-Venant model and for high order schemes.

\section{REFERENCES}

[1] S. Atzeni, J. Meyer-Ter-Vehn, The physics of Inertial Fusion, Beam Plasma Interaction, Hydrodynamics, Hot Dense Matter, International Series of Monographs on Physics, Volume 125, Oxford Sciences Publications, Oxford University Press (2009).

[2] M. Baudin, C. Berthon, F. Coquel, R. Masson and Q.H. Tran, A relaxation method for two flow models with hydrodynamic closure law, Numerische Mathematik, Volume 99, 411 (2005).

[3] C. Berthon, Inégalité d'entropie pour un schéma de relaxation, C. R., Acad. Sci. Paris, Ser. I, 340 : 63-68 (2005).

[4] C. Berthon, Stability of the muscl schemes for the Euler equations, Comm. Math. Sciences, Volume 3, 133 (2005).

[5] C. Berthon, Numerical approximations of the 10-moment Gaussian closure, Math. Comp., 75(256):1809-1831 (electronic) (2006).

[6] C. Berthon, P. Charrier, B. Dubroca, Asymptotic preserving relaxation scheme for a moment model of radiative transfer, C. R., Acad. Sci. Paris, Ser. I, 344 : 467-472 (2007).

[7] C. Berthon, B. Dubroca, A. Sangam, A local entropy minimum principle for deriving entropy preserving schemes, SIAM Journal of Numerical Analysis, Volume 50, 468-491 (2012).

[8] F. Bouchut, Entropy satisfying flux vector splittings and kinetic BGK models, Numerische Mathematik, Volume 94, 623 (2003).

[9] F. Bouchut, Nonlinear stability of finite volume methods for hyperbolic conservation laws, and well-balanced schemes for sources, Frontiers in Mathematics series, Birkhäuser (2004).

[10] S.L. Brown, P.L. Roe and C.P. Groth,Numerical Solution of a 10-Moment Model for Nonequilibrium Gasdynamics, 12th AIAA Computational Fluid Dynamics Conference (1995).

[11] P. Cargo, A.-Y. Leroux, Un schéma numérique adapaté au modèle d'atmosphère avec termes de gravité, C. R., Acad. Sci. Paris, Ser. I, 318 : 73-76 (1994).

[12] C. Chalons, Bilan d'entropie discrets dans l'approximation numérique des chocs non classics. Application aux équations de N.S multi-pression $2 D$ et à systèmes visco-capillaires, PhD thesis, École polytechnique (2002).

[13] C. Chalons and F. Coquel, Navier-Stokes equations with several independant pressure laws and explicit predictor-corrector schemes, Numerisch Math., Volume 103, 451-478 (2005)

[14] C. Chalons, F. Coquel, E. Godlewski, P.-A. Raviart, N. Seguin, Godunov-type schemes for hyperbolic systems with parameter-dependent source: the case of Euler system with friction, Math. Models Methods Appl. Sci., Volume 20, 2109 (2010).

[15] G.Q. Chen, C.D. Levermore and T.P. Liu, Hyperbolic Conservation Laws with Stiff Relaxation Terms and Entropy, Communication in Pure and Applied Mathematics, Volume 47, 787 (1995).

[16] F. Coquel and P. Perthame, Relaxation of Energy and Approximate Riemann Solver for General Pressure Laws in Fluids Dynamics, SIAM Journal of Numerical Analysis, Volume 35, 2223 (1998).

[17] R. P. Drake, High-Energy-Density Physics, Fundamentals, Inertial Fusion, and Experimental Astrophysics, Shock Wave and High Pressure Phenomena, Springer, Berlin, Heidelberg (2006).

[18] B. Dubroca, M. Tchong, P. Charrier, V.T. Tikhonchuk and J.-P. Morreeuw, Magnetic field generation in plasmas due to anisotropic laser heating, Physics of Plasmas, Volume 11, 3830 (2004).

[19] J.-L. Feugeas, Etude numérique des systèmes aux moments de Lervermore pour la modélisation d'écoulements bidimensionnels hors équilibre cinétique, PhD thesis, Université Bordeaux 1 (1997).

[20] E. Godlewski and P.-A. Raviart, Numerical Approximation of Hyperbolic Systems of Conservation Laws, Applied Mathematical Sciences, Volume 118, Springer, New York (1996).

[21] A. Harten, P.D. Lax, B. Van Leer, On Upstream Differencing and Godunov-Type Schemes for Hyperbolic Conservation Laws, SIAM Review, Volume 25, 35 (1983).

[22] S. Jin and Z. Xin, The Relaxation Schemes for Systems of Conservation Laws in Arbitrary Dimensions, Communication in Pure and Applied Mathematics, Volume 25, 235 (1995).

[23] W.L. Kruer, The Physics of Laser Plasma Interactions, Frontiers in Physics, Volume 73, Westview Press, Colorado (2003).

[24] P.D. Lax, Hyperbolic Systems of Conservation Laws and Mathematical Theory of Shock Waves, Conference Board of Mathematical Sciences, Conference Series in Applied Mathematics, SIAM, Philadelphia, Volume 11 (1973). 
[25] R.J. Leveque, Finite Volume Methods for Hyperbolic Problems, Cambridge Texts in Applied Mathematics, Cambridge University Press, Cambridge (2003).

[26] C.D. Levermore, Moment Closure Hierarchies for Kinetic Theories, Journal of Statistical Physics, Volume 83, 1021 (1996).

[27] C.D. Levermore and W.J. Morokoff, The Gaussian Moment Closure for Gas Dynamics, SIAM Journal of Applied Mathematics, Volume 59, 72 (1996).

[28] T.P. Liu, Hyperbolic Conservation Laws with Relation, Communication in Pure and Applied Mathematics, Volume 108, 153 (1987).

[29] J.-P. Morreeuw, A. Sangam, B. Dubroca, P. Charrier and V.T. Tikhonchuk, Electron temperature anisotropy modeling and its effect on anisotropy-magnetic field coupling in an underdense laser heated plasma, Journal de Physique IV, Volume 133, 295 (2006).

[30] A. Sangam, J.-P. Morreeuw, V.T. Tikhonchuk, Anisotropic instability in a laser heated plasma, Physics of Plasmas, Volume 14, 053111 (2007).

[31] A. Sangam, An HLLC Scheme for Ten-Moments coupled to Magnetic field, International Journal of Computing Sciences and Mathematics, Volume 2, 73 (2008).

[32] I. Suliciu, Energy estimates in rate-type thermo-viscoplasticity, Int. J. of Plast, Volume 14, 78 (1987)

[33] E. Tadmor, A minimum entropy principle in the gas dynamics equations, Applied Numerical Mathematics, Volume 2, 211 (1986).

[34] E.F. Toro, M. Spruce, W. Speares, Restoration of the contact surface in the HLL-Riemann solver, Shock Waves, Volume 4, 25 (1994).

[35] E.F. Toro, Riemann Solvers and Numerical Methods for Fluids dynamics, A Pratical Introduction, Third edition, Springer, Berlin (2009).

[36] Y. Xing and C.-W. Shu, High order well-balanced WENO scheme for the gas dynamics equations under gravitational fields, Journal of Scientific Computing, Volume 54, 645-662 (2013).

Appendix A. Rotational Invariance of the Flux Function and the Source

Term Function. Let $\mathcal{U}$ be the state variable as introduced in Section 2,

$$
\mathcal{U}=\left(\begin{array}{c}
\rho \\
\rho \boldsymbol{u} \\
\rho(\boldsymbol{u} \otimes \boldsymbol{u}+\varepsilon)
\end{array}\right) .
$$

Let us consider the expression of the directional flux function defined as follows:

$$
\mathbb{F}(\mathcal{U}, \boldsymbol{n})=(\boldsymbol{u} \cdot \boldsymbol{n}) \mathcal{U}+\left(\begin{array}{c}
0 \\
\rho \varepsilon \boldsymbol{n} \\
2((\rho \varepsilon \boldsymbol{n}) \otimes \boldsymbol{u})^{S}
\end{array}\right)
$$

where $\boldsymbol{n}$ denotes an arbitrary unit vector.

Let $R$ stands for a rotational matrix given for all $\theta \in(0,2 \pi)$ by,

$$
R=\left(\begin{array}{ccc}
\cos \theta & -\sin \theta & 0 \\
\sin \theta & \cos \theta & 0 \\
0 & 0 & 1
\end{array}\right) .
$$

We introduce the following rotational operator defined by

$$
\bar{R}(\mathcal{U})=\left(\begin{array}{c}
\rho \\
\rho R \boldsymbol{u} \\
\rho R(\boldsymbol{u} \otimes \boldsymbol{u}+\varepsilon) R^{T}
\end{array}\right) .
$$

Let us now check the relation

$$
\mathbb{F}(\bar{R}(\mathcal{U}), R \boldsymbol{n})=\bar{R}(\mathbb{F}(\mathcal{U}, \boldsymbol{n}))
$$


First, $\bar{R}(\mathbb{F}(\mathcal{U}, \boldsymbol{n}))$ writes,

$$
\begin{aligned}
\bar{R}(\mathbb{F}(\mathcal{U}, \boldsymbol{n})) & =(\boldsymbol{u} \cdot \boldsymbol{n}) \bar{R}(\mathcal{U})+\bar{R}\left(\begin{array}{c}
0 \\
\rho \boldsymbol{\varepsilon} \boldsymbol{n} \\
2((\rho \boldsymbol{\varepsilon} \boldsymbol{n}) \otimes \boldsymbol{u})^{S}
\end{array}\right), \\
& =(\boldsymbol{u} \cdot \boldsymbol{n}) \bar{R}(\mathcal{U})+\left(\begin{array}{c}
0 \\
\rho R(\boldsymbol{\varepsilon} \boldsymbol{n}) \\
2 R((\rho \boldsymbol{\varepsilon} \boldsymbol{n}) \otimes \boldsymbol{u})^{S} R^{T}
\end{array}\right),
\end{aligned}
$$

Second, $\mathbb{F}(\bar{R}(\mathcal{U}), R \boldsymbol{n})$ can be expressed as

$$
\left.\mathbb{F}(\bar{R}(\mathcal{U}), R \boldsymbol{n})=(R \boldsymbol{u} \cdot R \boldsymbol{n}) \bar{R}(\mathcal{U})+\left(\begin{array}{c}
0 \\
\rho\left(R \boldsymbol{\varepsilon} R^{T}\right) R \boldsymbol{n} \\
2\left(\left(\rho R\left(\varepsilon R^{T}\right) R \boldsymbol{n}\right) \otimes R \boldsymbol{u}\right.
\end{array}\right)^{S}\right)
$$

Since $R$ is a rotational matrix, the scalar product is invariant by $R$,

$$
R \boldsymbol{u} \cdot R \boldsymbol{n}=\boldsymbol{u} \cdot \boldsymbol{n} .
$$

Straightforward computations show that

$$
\left(R \boldsymbol{\varepsilon} R^{T}\right) R \boldsymbol{n}=R \boldsymbol{\varepsilon}\left(R^{T} R\right) \boldsymbol{n}=R(\boldsymbol{\varepsilon} \boldsymbol{n}) .
$$

Then we get

$$
\begin{aligned}
2\left(\left(\rho R \boldsymbol{\varepsilon} R^{T} \cdot R \boldsymbol{n}\right) \otimes R \boldsymbol{u}\right)^{S} & =2(\rho R(\boldsymbol{\varepsilon} \boldsymbol{n}) \otimes R \boldsymbol{u})^{S} \\
& =2 R((\rho \boldsymbol{\varepsilon} \boldsymbol{n}) \otimes \boldsymbol{u})^{S} R^{T}
\end{aligned}
$$

by direct computations.

Similar computations lead to invariance property of the source term $\mathbb{S}(\mathcal{U}, \nabla W, \boldsymbol{n})$ given by,

$$
\mathbb{S}(\mathcal{U}, \nabla W, \boldsymbol{n})=\left(\begin{array}{c}
0 \\
-\frac{1}{2} \rho(\boldsymbol{n} \otimes \nabla W) \boldsymbol{n} \\
-\rho(((\boldsymbol{n} \otimes \nabla W) \boldsymbol{n}) \otimes \boldsymbol{u})^{S}
\end{array}\right)
$$

Appendix B. Rotational Invariance of the Model (2.1). Let us consider the model (2.1),

$$
\left\{\begin{array}{l}
\partial_{t} \rho+\nabla_{x} \cdot(\rho \boldsymbol{u})=0 \\
\partial_{t}(\rho \boldsymbol{u})+\nabla_{x} \cdot(\rho \boldsymbol{u} \otimes \boldsymbol{u}+\boldsymbol{P})=-\frac{1}{2} \rho \boldsymbol{\nabla}_{x} W \\
\partial_{t}(\rho \boldsymbol{u} \otimes \boldsymbol{u}+\boldsymbol{P})+\nabla_{x} \cdot(\rho \boldsymbol{H} \otimes \boldsymbol{u})^{S}=-\frac{1}{2} \rho \boldsymbol{\nabla}_{x} W \otimes \boldsymbol{u}-\frac{1}{2} \rho \boldsymbol{u} \otimes \nabla_{x} W
\end{array}\right.
$$


where the subscript $x$ is added to emphasize that the corresponding operator is used according to coordinate $x$. Let $y, \boldsymbol{v}$ and $\boldsymbol{Q}$ be new coordinate, velocity and matrix pressure defined by

$$
\left\{\begin{array}{l}
y=R x \\
\boldsymbol{v}=R \boldsymbol{u} \\
\boldsymbol{Q}=R \boldsymbol{P} R^{T}
\end{array}\right.
$$

where $R$ stands for a rotational matrix given for all $\theta \in(0,2 \pi)$ by,

$$
R=\left(\begin{array}{ccc}
\cos \theta & -\sin \theta & 0 \\
\sin \theta & \cos \theta & 0 \\
0 & 0 & 1
\end{array}\right)
$$

We have,

$$
\left\{\begin{array}{l}
\boldsymbol{\nabla}_{x} \Psi=R^{T} \boldsymbol{\nabla}_{y} \Psi \\
\boldsymbol{\nabla}_{x} \cdot \boldsymbol{u}=\boldsymbol{\nabla}_{y} \cdot(R \boldsymbol{u})=\boldsymbol{\nabla}_{y} \cdot \boldsymbol{v} \\
\boldsymbol{\nabla}_{x} \cdot(\boldsymbol{T} R)=\boldsymbol{\nabla}_{y} \cdot \boldsymbol{T} \\
R \boldsymbol{\nabla}_{x} \cdot \boldsymbol{T}=\boldsymbol{\nabla}_{x} \cdot(R \boldsymbol{T})
\end{array}\right.
$$

where $\Psi$ and $\boldsymbol{T}$ are scalar and matrix, respectively.

According to (2.3), the first equation of (2.1) rewrites,

$$
0=\partial_{t} \rho+\nabla_{x} \cdot(\rho \boldsymbol{u})=\partial_{t} \rho+\nabla_{y} \cdot(\rho \boldsymbol{v}) .
$$

Multiplying the second equation of (2.1) by $R$ gives,

$$
\partial_{t}(\rho R \boldsymbol{u})+\nabla_{x} \cdot(R \rho \boldsymbol{u} \otimes \boldsymbol{u}+R \boldsymbol{P})=-\frac{1}{2} \rho R \nabla_{x} W,
$$

wich can be rewritten as

$$
\partial_{t}(\rho R \boldsymbol{u})+\nabla_{x} \cdot\left(R \rho \boldsymbol{u} \boldsymbol{u}^{T}+R \boldsymbol{P}\right)=-\frac{1}{2} \rho \nabla_{y} W,
$$

or

$$
\partial_{t}(\rho \boldsymbol{v})+\nabla_{x} \cdot\left(R \rho\left(R^{T} \boldsymbol{v}\right)\left(\boldsymbol{v}^{T} R\right)+R \boldsymbol{P} R^{T} R\right)=-\frac{1}{2} \rho \boldsymbol{\nabla}_{y} W
$$

that writes

$$
\partial_{t}(\rho \boldsymbol{v})+\nabla_{x} \cdot\left(\left(\rho \boldsymbol{v} \boldsymbol{v}^{T}+R \boldsymbol{P} R^{T}\right) R\right)=-\frac{1}{2} \rho \nabla_{y} W
$$

or equivalently,

$$
\partial_{t}(\rho \boldsymbol{v})+\nabla_{y} \cdot\left(\rho \boldsymbol{v} \boldsymbol{v}^{T}+R \boldsymbol{P} R^{T}\right)=-\frac{1}{2} \rho \nabla_{y} W .
$$

This takes the following form,

$$
\partial_{t}(\rho \boldsymbol{v})+\nabla_{y} \cdot(\rho \boldsymbol{v} \otimes \boldsymbol{v}+\boldsymbol{Q})=-\frac{1}{2} \rho \nabla_{y} W .
$$


Note that the following identity holds,

$$
\nabla_{y} \cdot \boldsymbol{Q}=\nabla_{y} \cdot\left(R \boldsymbol{P} R^{T}\right)=\nabla_{y} \cdot \boldsymbol{P}
$$

The last equation of (2.1) can be rewritten as

$$
\begin{aligned}
\partial_{t}\left(R^{T}\left(\rho \boldsymbol{v} \otimes \boldsymbol{v}+R \boldsymbol{P} R^{T}\right) R\right)+\nabla_{x} \cdot(\rho \boldsymbol{H} \otimes \boldsymbol{u})^{S} & = \\
& \quad-\frac{1}{2} \rho \boldsymbol{\nabla}_{x} W \otimes \boldsymbol{v} R-\frac{1}{2} \rho R^{T} \boldsymbol{v} \otimes \nabla_{x} W,
\end{aligned}
$$

or

$$
\begin{aligned}
\partial_{t}\left(\rho \boldsymbol{v} \otimes \boldsymbol{v}+R \boldsymbol{P} R^{T}\right)+ & R\left(\boldsymbol{\nabla}_{x} \cdot(\rho \boldsymbol{H} \otimes \boldsymbol{u})^{S}\right) R^{T}= \\
& -\frac{1}{2} \rho R \boldsymbol{\nabla}_{x} W \otimes \boldsymbol{v}-\frac{1}{2} \rho R^{T} \boldsymbol{v} \otimes \boldsymbol{\nabla}_{x} W R^{T} .
\end{aligned}
$$

We have

$$
\left\{\begin{array}{l}
R \boldsymbol{\nabla}_{x} W \otimes \boldsymbol{v}=R \boldsymbol{\nabla}_{x} W \boldsymbol{v}^{T}=\left(R \boldsymbol{\nabla}_{x} W\right) \boldsymbol{v}^{T}=\boldsymbol{\nabla}_{y} W \boldsymbol{v}^{T}=\boldsymbol{\nabla}_{y} W \otimes \boldsymbol{v}, \\
\boldsymbol{v} \otimes \boldsymbol{\nabla}_{x} W R^{T}=\boldsymbol{v}\left(\boldsymbol{\nabla}_{x} W\right)^{T} R^{T}=\boldsymbol{v}\left(R \boldsymbol{\nabla}_{x} W\right)^{T}=\boldsymbol{v}\left(\boldsymbol{\nabla}_{y} W\right)^{T}=\boldsymbol{v} \otimes \nabla_{y} W .
\end{array}\right.
$$

The tensor $\mathcal{H}=R \boldsymbol{H} R^{T}=R(\boldsymbol{u} \otimes \boldsymbol{u}+3 \boldsymbol{P} / \rho) R^{T}=\left((R \boldsymbol{u}) \otimes(R \boldsymbol{u})+3 R \boldsymbol{P} R^{T} / \rho\right)=\boldsymbol{v} \otimes \boldsymbol{v}+$ $3 \boldsymbol{Q} / \rho$ can be understood as the generalized enthalpy $\boldsymbol{H}$ expressed in the new frame. Hence, the flux of tensor energy equation can be expressed as,

$$
R\left(\boldsymbol{\nabla}_{x} \cdot(\rho \boldsymbol{H} \otimes \boldsymbol{u})^{S}\right) R^{T}=\left(\boldsymbol{\nabla}_{x} \cdot\left(\rho\left(R \boldsymbol{H} R^{T}\right) \otimes(R \boldsymbol{u})\right)^{S}\right) R^{T}=\boldsymbol{\nabla}_{y} \cdot(\rho \mathcal{H} \otimes \boldsymbol{v})^{S} .
$$

Therefore, the last equation of (2.1) can be rewritten as

$$
\partial_{t}(\rho \boldsymbol{v} \otimes \boldsymbol{v}+\boldsymbol{Q})+\nabla_{y} \cdot(\rho \mathcal{H} \otimes \boldsymbol{v})^{S}=-\frac{1}{2} \rho \nabla_{y} W \otimes \boldsymbol{v}-\frac{1}{2} \rho \boldsymbol{v} \otimes \nabla_{y} W
$$

and the Ten-Moments endows the rotationnal invariance since in the rotated frame along the definitions (2.2), it takes the following shape,

$$
\left\{\begin{array}{l}
\partial_{t} \rho+\nabla_{y} \cdot(\rho \boldsymbol{u})=0, \\
\partial_{t}(\rho \boldsymbol{v})+\nabla_{y} \cdot(\rho \boldsymbol{v} \otimes \boldsymbol{v}+\boldsymbol{Q})=-\frac{1}{2} \rho \boldsymbol{\nabla}_{y} W, \\
\partial_{t}(\rho \boldsymbol{v} \otimes \boldsymbol{v}+\boldsymbol{Q})+\nabla_{y} \cdot(\rho \mathcal{H} \otimes \boldsymbol{v})^{S}=-\frac{1}{2} \rho \boldsymbol{\nabla}_{y} W \otimes \boldsymbol{v}-\frac{1}{2} \rho \boldsymbol{v} \otimes \nabla_{y} W .
\end{array}\right.
$$



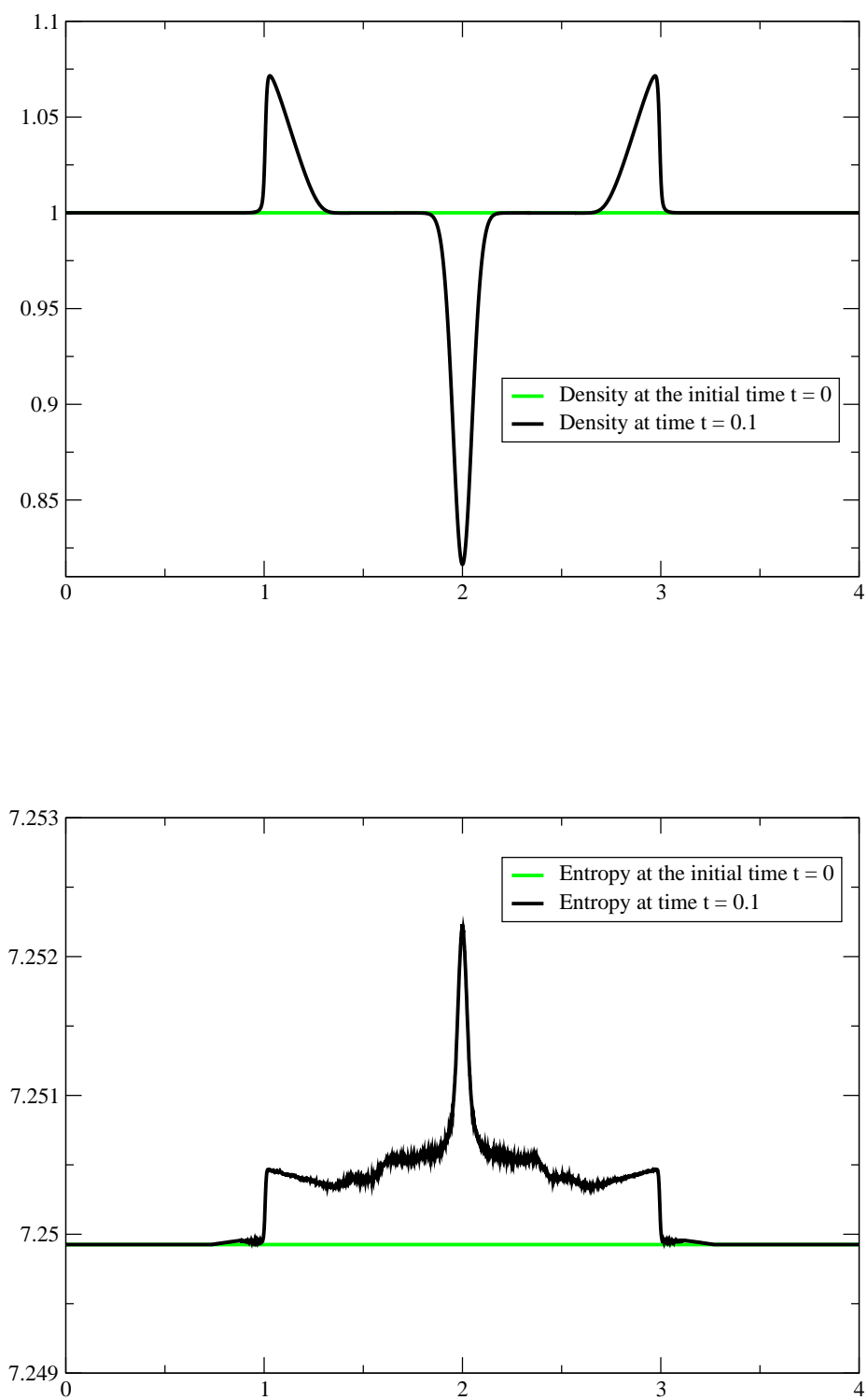

FIG. 7.1. Uniform plasma state with a gaussian-type source term: density $\rho$ calculated at time 0.1 , and entropy s computed at times 0 and 0.1 by the relaxation scheme proposed in this work. 

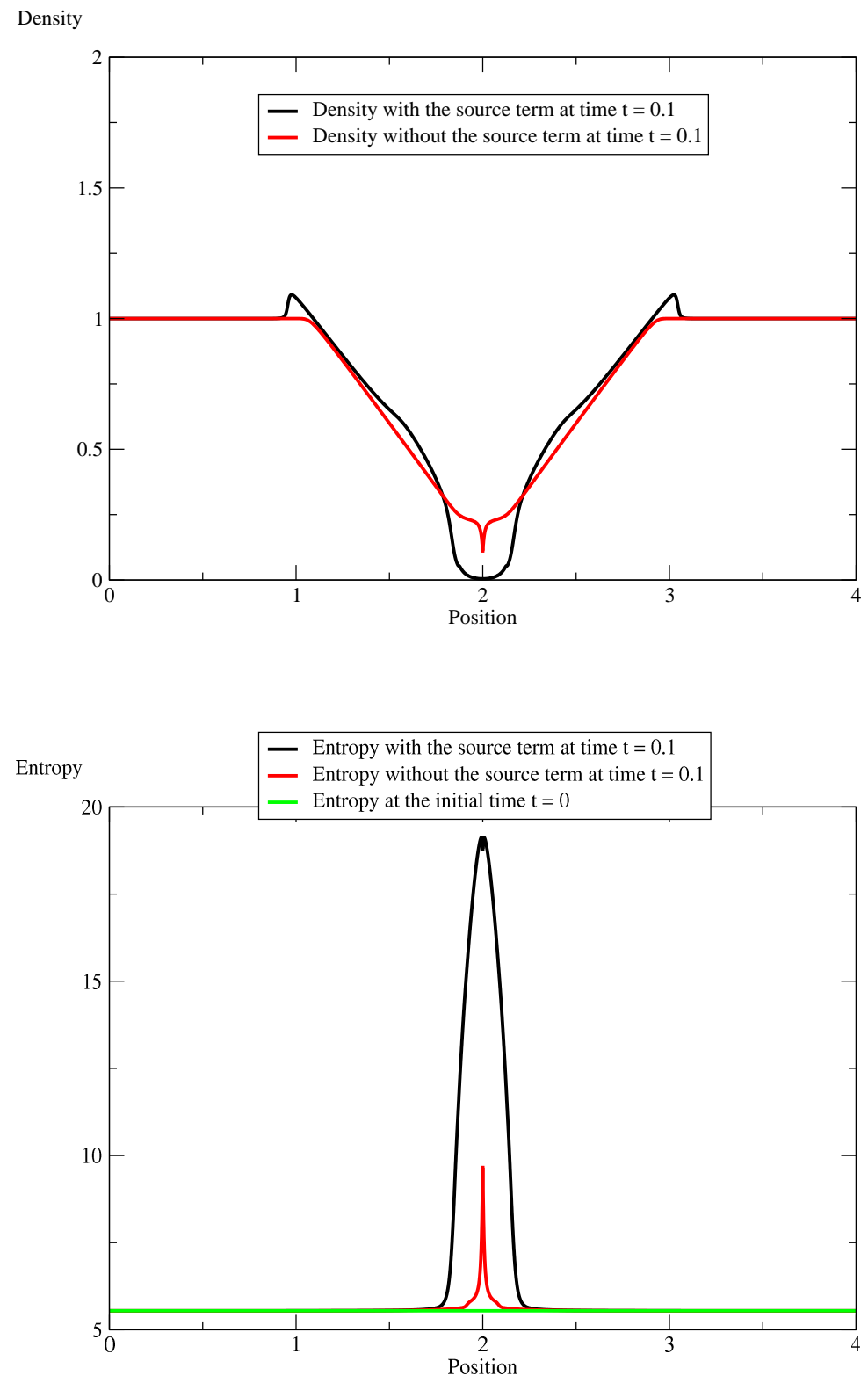

FIG. 7.2. Two rarefaction waves problem with and without a gaussian-type source term: density $\rho$ computed at time 0.05 , and entropy s computed at times 0 and 0.1 by the relaxation scheme proposed in this work. 


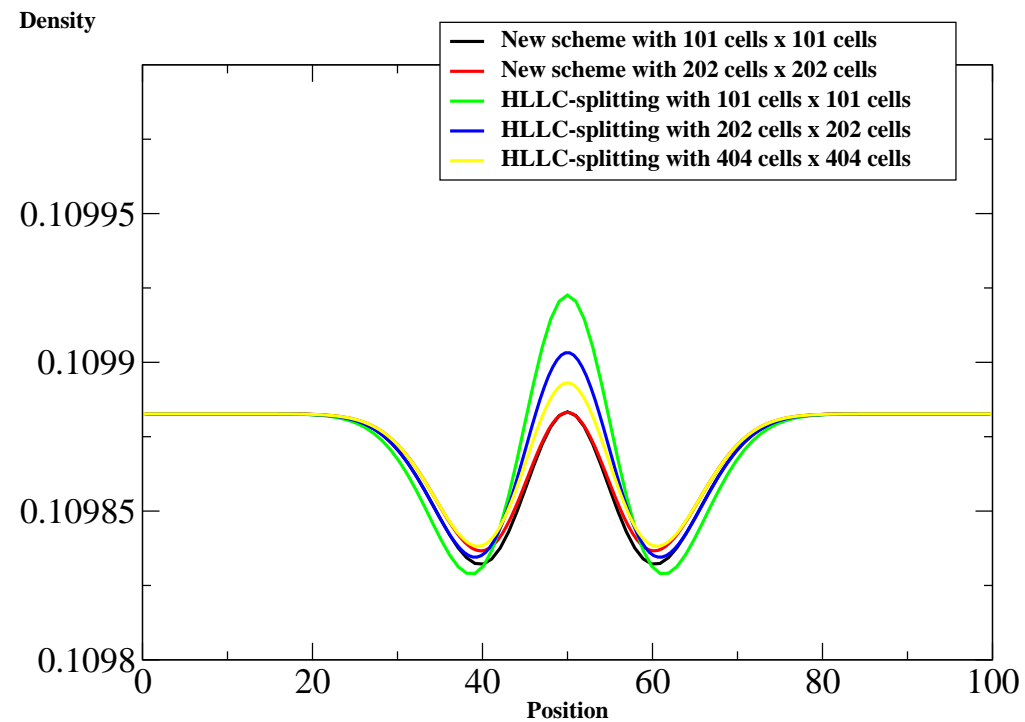

FIG. 7.3. Two dimensional test problem of laser-matter interaction: density $\rho$ computed at time 5 ps by the relaxation scheme proposed in this work. The comparisons are also proposed with the solutions rendered by the HLLC-splitting scheme. 

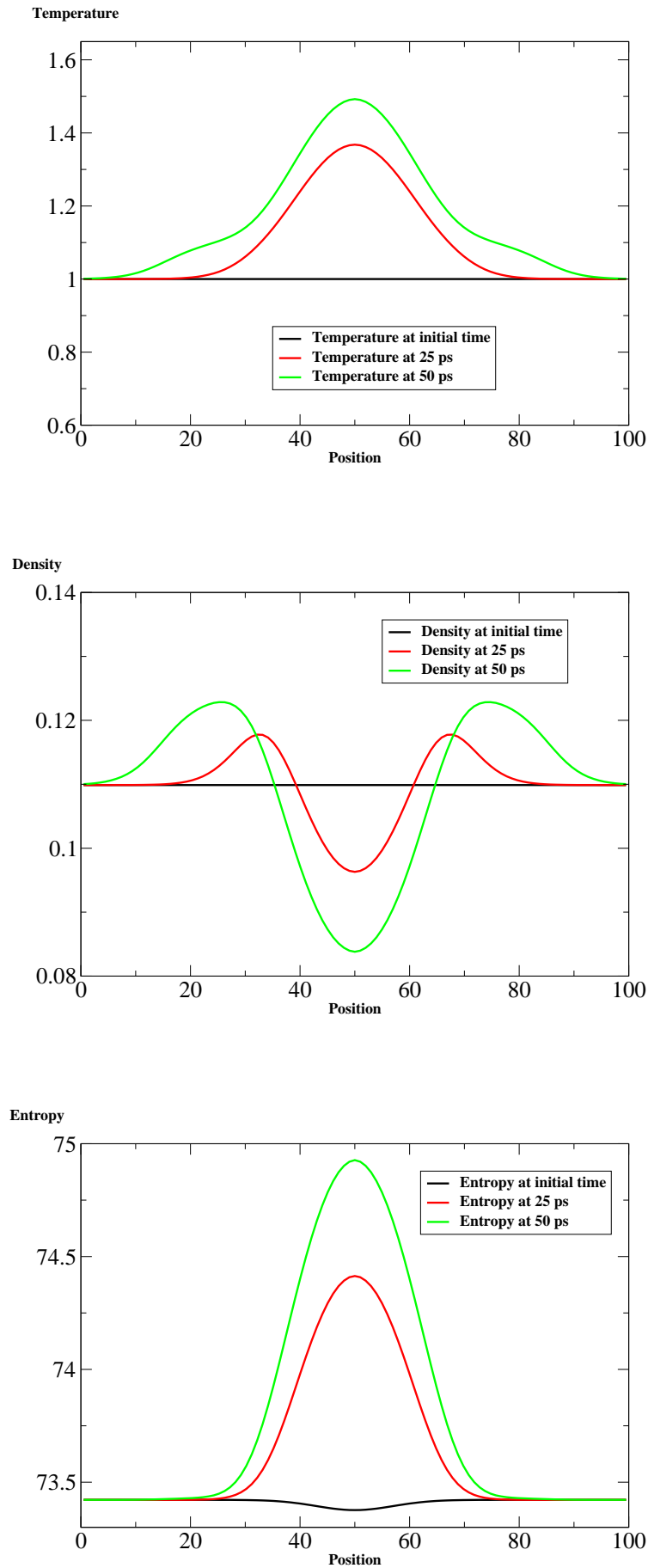

FIG. 7.4. Two dimensional test problem of laser-matter interaction taking to account the inverse Bremsstrahlung effect. Temperature, density $\rho$ and entropy are computed at the initial time, at times $25 \mathrm{ps}, 50 \mathrm{ps}$ by the relaxation scheme proposed in this work. Temperature is scaled at its the initial value $T_{e_{0}}=2.3 \times 10^{7} \mathrm{~K}$ while density is scaled at $n_{e_{0}} m_{i} / Z=10^{27} m_{i} / 4$ where $m_{i}$ is the ion mass. 


\section{Anisotropy}

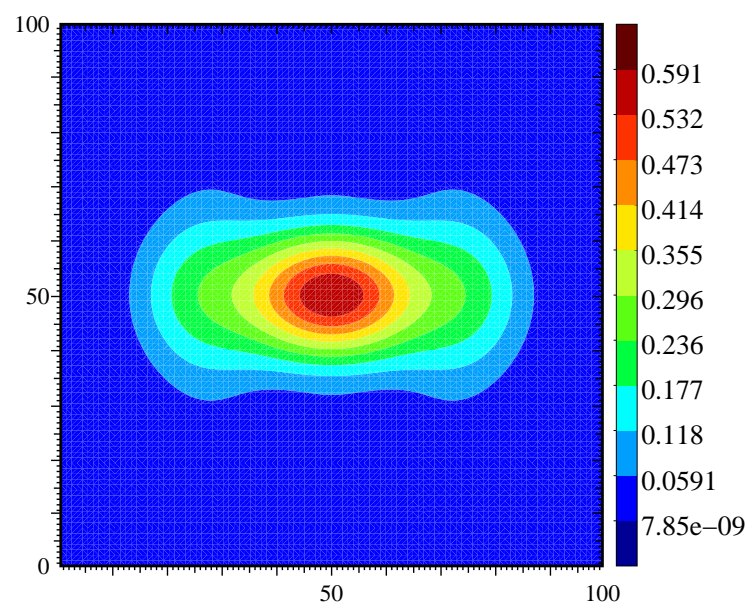

Temperature

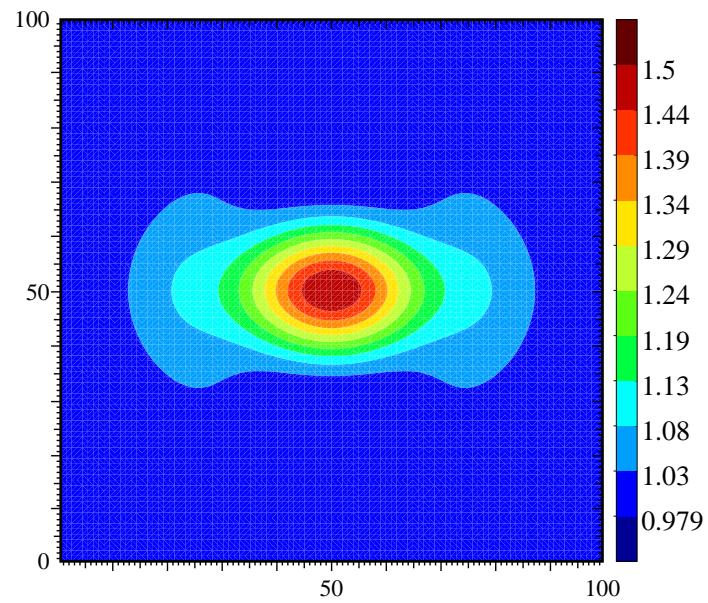

Density

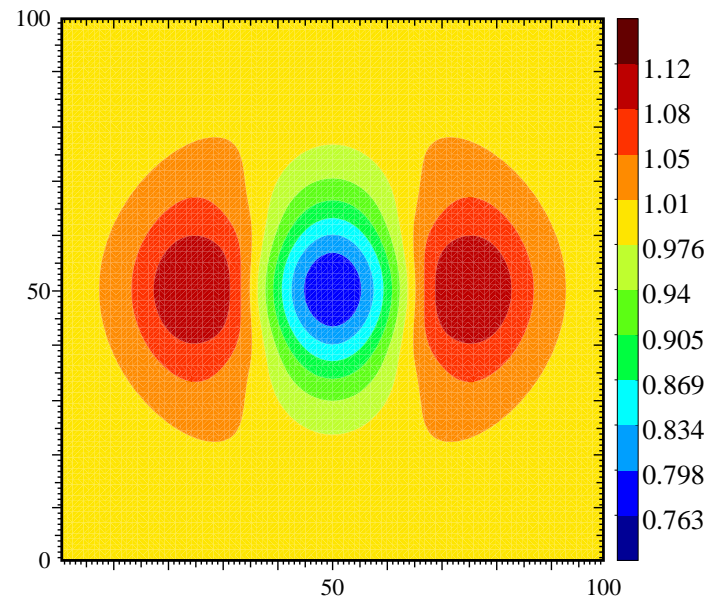

FIG. 7.5. Two dimensional test problem of laser-matter interaction taking to account the inverse bremsstrahlung effect. Temperature, density $\rho$ and entropy are computed at 50 ps by the relaxation scheme proposed in this work. Temperature is scaled at its the initial value $T_{e_{0}}=2.3 \times 10^{7} \mathrm{~K}$ while density is scaled at $n_{e_{0}} m_{i} / Z=10^{27} m_{i} / 4$ where $m_{i}$ is the ion mass. 